\title{
VEGAS: A VST Early-type GAlaxy Survey
}

\section{Photometric study of giant ellipticals and their stellar halos}

\author{
Marilena Spavone ${ }^{1}$, Massimo Capaccioli ${ }^{1,2}$, Nicola R. Napolitano ${ }^{1}$, Enrichetta Iodice ${ }^{1}$, \\ Aniello Grado ${ }^{1}$, Luca Limatola ${ }^{1}$, Andrew P. Cooper ${ }^{4}$, Michele Cantiello ${ }^{1,3}$, Duncan A. Forbes ${ }^{5}$, \\ Maurizio Paolillo ${ }^{2}$, and Pietro Schipani ${ }^{1}$
}

\footnotetext{
1 INAF-Astronomical Observatory of Capodimonte, Salita Moiariello 16, 80131 Naples, Italy e-mail: spavone@na.astro.it

2 University of Naples Federico II, C.U. Monte Sant'Angelo, via Cinthia, 80126 Naples, Italy

3 INAF-Astronomical Observatory of Teramo, via Maggini, 64100 Teramo, Italy

4 Institute for Computational Cosmology, Durham DH13, UK

5 Centre for Astrophysics \& Supercomputing, Swinburne University, Hawthorn, VIC 3122, Australia
}

Received 14 June 2016 / Accepted 30 March 2017

\begin{abstract}
Observations of diffuse starlight in the outskirts of galaxies are thought to be a fundamental source of constraint on the cosmological context of galaxy assembly in the $\Lambda \mathrm{CDM}$ model. Such observations are not trivial because of the extreme faintness of such regions. In this work, we investigated the photometric properties of six massive early-type galaxies (ETGs) in the VEGAS sample (NGC 1399, NGC 3923, NGC 4365, NGC 4472, NGC 5044, and NGC 5846) out to extremely low surface brightness levels with the goal of characterizing the global structure of their light profiles for comparison to state-of-the-art galaxy formation models. We carried out deep and detailed photometric mapping of our ETG sample taking advantage of deep imaging with VST/OmegaCAM in the $g$ and $i$ bands. By fitting the light profiles, and comparing the results to simulations of elliptical galaxy assembly, we have identified signatures of a transition between relaxed and unrelaxed accreted components and can constrain the balance between in situ and accreted stars. The very good agreement of our results with predictions from theoretical simulations demonstrates that the full VEGAS sample of $\sim 100$ ETGs will allow us to use the distribution of diffuse light as a robust statistical probe of the hierarchical assembly of massive galaxies.
\end{abstract}

Key words. galaxies: elliptical and lenticular, $\mathrm{cD}$ - galaxies: fundamental parameters - galaxies: formation - galaxies: halos techniques: image processing

\section{Introduction}

The cold dark matter (LCDM) galaxy formation theories predict that galaxies grow through a combination of in situ star formation and accretion of stars from other galaxies (White \& Frenk 1991). The ratio of stellar mass contributed by these two modes of growth is expected to change systematically over the lifetime of a galaxy as its dark matter halo and star formation efficiency evolve (e.g. Guo \& White 2008). Accreted stars are expected to dominate in the outer parts of galaxies because they have much lower binding energies in the remnant system than stars formed by dissipative collapse. Since dynamical timescales are long in these outer regions, phase-space substructures related to accretion, such as streams and caustics, can persist over many gigayears.

The structural properties of the outer parts of galaxies and their correlations with stellar mass and other observables might therefore provide ways of testing theoretical predictions of growth by accretion. For example, they could constrain the overall ratio of stars accreted to those formed in situ, the number of significant progenitors, and the dynamics of the most important merger events (Johnston et al. 2008; Cooper et al. 2010; Deason et al. 2013; Amorisco 2017). In support of this idea, cosmological simulations of galaxy formation predict trends in these quantities with mass that are responsible for systematic variations in the average shape, amplitude, and extent of azimuthally averaged surface brightness profiles (Font et al. 2011; Cooper et al. 2013; Pillepich et al. 2014). Unfortunately, the accreted debris is also expected to have extremely low surface brightness $\left(\mu_{V} \sim 29 \mathrm{mag} / \operatorname{arcsec}^{2}\right)$ on average and therefore to be very hard to separate from the background sky in conventional photometric observations.

In this paper we use extremely deep images of six massive early-type galaxies (ETGs) from the VEGAS survey (described below) to constrain the properties of their accreted stellar components. The brightest ETGs are thought to be predominantly the central galaxies of the most massive DM halos at the present day (e.g. Frenk et al. 1985; Kauffmann et al. 1993; De Lucia et al. 2006). In order to explain the exponential highmass cut-off of the galactic stellar mass function, theoretical models have invoked processes whereby in situ star formation in such halos is strongly suppressed at late times (e.g. Bower et al. 2006). As a consequence, massive ETGs in these models accumulate the bulk of their stellar mass by accretion, predominantly through $\sim 10$ mergers (in the case of massive BCGs; De Lucia \& Blaizot 2007; Cooper et al. 2015) of low stellar mass ratio. Their spheroidal morphology is thought to arise from violent relaxation of the central potential during the most massive of these mergers, which can erase coherent structures established by earlier dissipative star formation 
(Villumsen 1983; Cole et al. 2000; Hilz et al. 2012). The same models predict a rather different relationship between mass growth and structure for late-type galaxies (LTGs) in less massive dark matter halos, such as the Milky Way. In LTGs, the vast majority of stars are formed in situ in rotating discs that survive to the present day, while the bulk of the accreted stars are contributed by one or two objects of much lower mass and are mostly deposited on weakly bound orbits extending far from the centre of the potential. These models therefore imply a natural but complex continuum between the properties of the dominant stellar components of ETGs and those of the diffuse spheroidal "stellar halos" around LTGs such as the Milky Way, all of which originate from the same hierarchical accretion process (Purcell et al. 2007; Cooper et al. 2013).

From an observational perspective, it is straightforward to separate the disc and stellar halo components of LTGs because their mass, kinematics, spatial distribution, and stellar populations are very different. Moreover, with relatively little ambiguity, the empirically defined stellar halo can be identified with the bulk of the accreted stellar mass in those galaxies and the disc with the bulk of in situ stellar mass. In ETGs, however, the connections between different mechanisms of mass growth and the "structural components" inferred from images are much less straightforward. If the bulk of the stars are really accreted, then the "stellar halo" (or "spheroid" or "classical bulge") should be identified with at least the structural component that dominates the observed stellar mass. However, other empirical "components" might also be accreted. In situ stars in ETGs are extremely difficult to distinguish if they are also spheroidal, dispersionsupported and have old, metal-rich stellar populations resembling those of the dominant accreted component(s) with which they have been thoroughly mixed by violent relaxation ${ }^{1}$.

Cosmological dynamical simulations can help by suggesting plausible interpretations for features in the surface brightness profiles of ETGs in the context of specific galaxy formation theories. In particular, simulated galaxies show evidence of substructure in the form of inflections ("breaks"), at which the surface brightness profile either becomes steeper or shallower (e.g. Cooper et al. 2013; Rodriguez-Gomez et al. 2016). As noted above, models predict that the accreted components of ETGs comprise several equal-size chunks of stellar mass from different contributors, but also that these contributors have a range of relative total (virial) mass ratios, such that their debris relax to distributions with a range of characteristic radii (Amorisco 2017). As shown for example in Fig. 10 of Cooper et al. (2015), inflections in the profile may therefore correspond to the transition between in situ and accretion dominance, which is responsible for the most obvious profile inflection for most LTGs, although this occurs at relatively low surface brightness (SB) (Abadi et al. 2006; Font et al. 2011; Pillepich et al. 2014; Rodriguez-Gomez et al. 2016) More generally, these inflections also correspond to variations in the ratio between individual accreted components as a function of radius (Cooper et al. 2010; Deason et al. 2013; Amorisco 2017). Indeed, for the reasons given above, most simulations ${ }^{2}$ predict that the transition between in situ and accretiondominated regions should be almost imperceptible in the azimuthally averaged profiles of typical ETGs, even though it

\footnotetext{
1 In the extreme case of a merger between two identical elliptical galaxies in identical DM halos, it would clearly be impossible to differentiate between the in situ component and the "accreted" component in the surface brightness profile of the remnant.

2 The exception is simulations that do not strongly suppress in situ star formation in very massive galaxies, which are generally in disagreement with observed luminosity functions.
}

occurs at relatively high SB. This transition may still be detectable as a change in shape or stellar population.

Observational work has clearly identified features in ETG surface brightness profiles and kinematic distributions that are indicative of substructure in the accreted component (Bender et al. 2015; Longobardi et al. 2015a,b; Foster et al. 2016). Some of these feature occur at distances of many effective radii and hence at very low SB, which could be interpreted as evidence for multiple accreted components in the context of the models mentioned above. Using different techniques with observations of different depths, several authors have concluded that the profiles of massive ETGs are not well described by a single Sérsic $r^{1 / n}$ law component, once thought to be near universal for spheroidal galaxies. For example, Gonzalez et al. (2005) found that the surface brightness profiles of BCGs in their sample were well fitted by a double $r^{1 / 4}$ de Vaucouleurs law, while other works have argued that a combination of a Sérsic and an exponential $(n=1)$ component provide the best description of particular ETG light profiles (Seigar et al. 2007; Donzelli et al. 2011; Bender et al. 2015). Huang et al. (2013) have suggested that three or more Sérsic components may be required for an accurate description of typical ETGs.

A recent revival of this topic, historically hampered by the faintness of galaxian halos (de Vaucouleurs \& Capaccioli 1979; D'Onofrio et al. 1994), has been fostered by the forthcoming operation of a new generation of very-wide-field imaging instruments (Ferrarese et al. 2012; Mihos et al. 2013; Duc et al. 2015; Capaccioli et al. 2015; Iodice et al. 2016). We are taking advantage of the wide field of view and high spatial resolution of the VLT Survey Telescope (VST; Capaccioli \& Schipani 2011) at the ESO Cerro Paranal Observatory (Chile) to carry out a multiband imaging survey of nearby ETGs, named VEGAS (VST Early-type GAlaxies Survey) (Capaccioli et al. 2015; hereafter Paper I). The primary aim of the survey is to obtain maps of the surface brightness of galaxies with heliocentric redshifts $V_{\text {rad }}<4000 \mathrm{~km} \mathrm{~s}^{-1}$ and $M_{B_{\text {tot }}}<-19.2$ mag sufficiently deep to detect the signatures of diffuse stellar components; the expected depths are $\mu_{g} \sim 27.3, \mu_{\mathrm{r}} \sim 26.8$, and $\mu_{i} \sim 26 \mathrm{mag} / \mathrm{arcsec}^{2}$. The large, homogeneous, and deep sample from VEGAS will enable a more systematic analysis of ETG light profiles and other photometric trends with radius than has previously been possible. We expect to obtain deep images for a sample of 100 ETGs across a range of environments. To date, we have collected data for about 30 galaxies. The present status of VEGAS observations is regularly updated at the link http://www.na.astro. it/vegas/VEGAS/VEGAS_Targets.html.

The subsample of VEGAS galaxies that we study here are listed in Table 1 together with their basic parameters. These galaxies were chosen because of their large angular dimensions, and because they are the brightest galaxies in their local environment. Here we present and analyse the $g$ - and $i$-band photometric properties of the four galaxies NGC 4365, NGC 3923, NGC 5044, and NGC 5846, which are in small groups and clusters. We combine these results with those presented in Paper I for NGC 4472 in the Virgo cluster, and in Iodice et al. (2016) for NGC 1399 in the Fornax cluster. Since both NGC 4472 and NGC 1399 are targets of the VEGAS survey and have been observed with VST, together with the other objects they represent a homogeneous sample of bright ETGs.

The paper is organized as follows. In Sect. 2 we describe the galaxies in our sample and their main properties. The observing strategy and the data reduction procedure are described in Sect. 3, while the data analysis is presented in Sects. 4 and 5. In 
Table 1. Basic properties of the galaxies studied in this paper.

\begin{tabular}{|c|c|c|}
\hline Parameter & Value & Ref. \\
\hline \multicolumn{3}{|c|}{ NGC 1399} \\
\hline $\begin{array}{l}\text { Morphological type } \\
\text { RA (J2000) } \\
\text { Dec (J2000) } \\
\text { Helio. radial velocity } \\
\text { Distance } \\
\text { Absolute magnitude } M_{g}\end{array}$ & $\begin{array}{c}\mathrm{E} 1 \\
03^{\mathrm{h}} 38^{\mathrm{m}} 29.0^{\mathrm{s}} \\
-35^{\mathrm{d}} 27^{\mathrm{m}} 02^{\mathrm{s}} \\
1425 \mathrm{~km} \mathrm{~s}^{-1} \\
20.9 \mathrm{Mpc}^{-23.05^{b}} \\
-2\end{array}$ & $\begin{array}{c}\text { RC3 } \\
\text { NED } \\
\text { NED } \\
\text { NED } \\
\text { Blakeslee et al. (2009) } \\
\text { Iodice et al. (2016) } \\
\end{array}$ \\
\hline \multicolumn{3}{|c|}{ NGC 3923} \\
\hline $\begin{array}{l}\text { Morphological type } \\
\text { RA (J2000) } \\
\text { Dec (J2000) } \\
\text { Helio. radial velocity } \\
\text { Distance } \\
\text { Absolute magnitude } M_{g}\end{array}$ & $\begin{array}{c}\mathrm{E} 4 \\
11^{\mathrm{h}} 51^{\mathrm{m}} 01.7^{\mathrm{s}} \\
-28^{\mathrm{d}} 48^{\mathrm{m}} 22^{\mathrm{s}} \\
1739 \mathrm{~km} \mathrm{~s}^{-1} \\
22.9 \mathrm{Mpc} \\
-22.66^{b} \\
\end{array}$ & $\begin{array}{c}\text { RC3 } \\
\text { NED } \\
\text { NED } \\
\text { NED } \\
\text { Tonry et al. (2001) } \\
\text { This work } \\
\end{array}$ \\
\hline \multicolumn{3}{|c|}{$N G C 4365$} \\
\hline $\begin{array}{l}\text { Morphological type } \\
\text { RA (J2000) } \\
\text { Dec (J2000) } \\
\text { Helio. radial velocity } \\
\text { Distance } \\
\text { Absolute magnitude } M_{g} \\
\end{array}$ & $\begin{array}{c}\mathrm{E} 3 \\
12^{\mathrm{h}} 24^{\mathrm{m}} 28.3^{\mathrm{s}} \\
+07^{\mathrm{d}} 19^{\mathrm{m}} 04^{\mathrm{s}} \\
1243 \mathrm{~km} \mathrm{~s}^{-1} \\
23.1 \mathrm{Mpc}^{-22.41^{a}} \\
\end{array}$ & $\begin{array}{c}\text { RC3 } \\
\text { NED } \\
\text { NED } \\
\text { NED } \\
\text { Blakeslee et al. (2009) } \\
\text { This work } \\
\end{array}$ \\
\hline \multicolumn{3}{|c|}{$N G C 4472$} \\
\hline $\begin{array}{l}\text { Morphological type } \\
\text { RA (J2000) } \\
\text { Dec (J2000) } \\
\text { Helio. radial velocity } \\
\text { Distance } \\
\text { Absolute magnitude } M_{g}\end{array}$ & $\begin{array}{c}\mathrm{E} 2 \\
12^{\mathrm{h}} 29^{\mathrm{m}} 46.7^{\mathrm{s}} \\
+08^{\mathrm{d}} 00^{\mathrm{m}} 02^{\mathrm{s}} \\
981 \mathrm{~km} \mathrm{~s}^{-1} \\
16.9 \mathrm{Mpc} \\
-22.77^{a} \\
\end{array}$ & $\begin{array}{c}\text { RC3 } \\
\text { NED } \\
\text { NED } \\
\text { NED } \\
\text { Mei et al. (2007) } \\
\text { This work } \\
\end{array}$ \\
\hline \multicolumn{3}{|c|}{$N G C 5044$} \\
\hline $\begin{array}{l}\text { Morphological type } \\
\text { RA (J2000) } \\
\text { Dec (J2000) } \\
\text { Helio. radial velocity } \\
\text { Distance } \\
\text { Absolute magnitude } M_{g}\end{array}$ & $\begin{array}{c}\mathrm{E} 0 \\
13^{\mathrm{h}} 15^{\mathrm{m}} 24.0^{\mathrm{s}} \\
-16^{\mathrm{d}} 23^{\mathrm{m}} 08^{\mathrm{s}} \\
2782 \mathrm{~km} \mathrm{~s}^{-1} \\
31.19 \mathrm{Mpc} \\
-22.59^{b} \\
\end{array}$ & $\begin{array}{c}\text { RC3 } \\
\text { NED } \\
\text { NED } \\
\text { NED } \\
\text { Tonry et al. (2001) } \\
\text { This work } \\
\end{array}$ \\
\hline \multicolumn{3}{|c|}{ NGC 5846} \\
\hline $\begin{array}{l}\text { Morphological type } \\
\text { RA (J2000) } \\
\text { Dec (J2000) } \\
\text { Helio. radial velocity } \\
\text { Distance } \\
\text { Absolute magnitude } M_{g}\end{array}$ & $\begin{array}{c}\mathrm{E} 0 \\
15^{\mathrm{h}} 06^{\mathrm{m}} 29.3^{\mathrm{s}} \\
+01^{\mathrm{d}} 36^{\mathrm{m}} 20^{\mathrm{s}} \\
1712 \mathrm{~km} \mathrm{~s}^{-1} \\
24.89 \mathrm{Mpc} \\
-22.77^{b} \\
\end{array}$ & $\begin{array}{c}\text { RC3 } \\
\text { NED } \\
\text { NED } \\
\text { NED } \\
\text { Tonry et al. (2001) } \\
\text { This work } \\
\end{array}$ \\
\hline
\end{tabular}

Notes. (a) Corrected for interstellar extinction as in Arrigoni Battaia et al. (2012). (b) Corrected for interstellar extinction as in Burstein \& Heiles (1982).

Sect. 6 we compare our data with theoretical predictions, and in Sect. 7 we discuss our results and draw conclusions.

\section{The sample}

We briefly describe here the main properties of the four galaxies analyzed in this work: NGC 3923, NGC 4365, NGC 5044 and
NGC 5846, and those of NGC 4472 and NGC 1399 (published in Paper I and by Iodice et al. 2016, respectively).

\subsection{NGC 1399}

NGC 1399 (FCC 213) is the central and brightest elliptical galaxy in the Fornax cluster. It has been extensively studied in a wide range of wavelengths. This galaxy has a vast and diffuse stellar envelope surrounding it (Iodice et al. 2016) and an extended and asymmetric gaseous halo in X-rays (Paolillo et al. 2002). NGC 1399 is the subject of a paper by Iodice et al. (2016), in which its main properties are discussed at length.

\subsection{NGC 3923}

NGC 3923, first studied by Malin \& Carter (1980), is one of the best examples of a "shell galaxy", with a vast system of peculiar, concentric shells. Sandage \& Bedke (1994) found at least seven dwarf ellipticals in the field centred on NGC 3923, which is the brightest member of this small group. Carter et al. (1998) found that the galaxy has minor axis rotation. This could be either the result of a kinematically decoupled core, or evidence of a triaxial geometry. Buote \& Canizares (1998), by studying the X-ray isophotes of NGC 3923, found evidence of a dark matter distribution more flattened and extended than the optical luminosity distribution.

\subsection{NGC 4365}

NGC 4365 (VCC 0731) is a bright and giant elliptical galaxy located in a group slightly behind the "Virgo subcluster B" centred on NGC 4472. It shows the presence of a small scale disc in the centre that is bluer than the surrounding galaxy (Ferrarese et al. 2006).

Peng et al. (2006) detected a significant population of globular clusters (GCs) around this object, with a specific frequency higher than in other galaxies of comparable luminosity, probably due to a larger number of red GCs. Moreover, Blom et al. (2012a,b) found the GC system to consist of three subpopulations.

Surma (1993) found evidence for two kinematically distinct stellar components: a bulge-like component and a disc-like subsystem. The most interesting kinematic features of NGC 4365 are its kinematically decoupled core (Davies et al. 2001) and that the galaxy rolls rather than rotates (Arnold et al. 2014). Bogdán et al. (2012) found an extended faint tidal tail between NGC 4365 and NGC 4342 in deep B-band images $\left(\mu_{B} \sim\right.$ $29.5 \mathrm{mag} / \operatorname{arcsec}^{2}$ ), which Blom et al. (2014) claimed to be a sign of ongoing interaction between the two galaxies.

\subsection{NGC 4472}

NGC 4472 (M49), a giant elliptical galaxy, is the brightest member of the Virgo cluster. It lies in the centre of the southern grouping of the cluster, also known as "Virgo subcluster B". This galaxy has been extensively studied by many authors (see e.g. Kim et al. 2000; Ferrarese et al. 2006; Kormendy et al. 2009; Janowiecki et al. 2010; Mihos et al. 2013) and, together with its companion galaxies, it was the subject of the first VEGAS paper (Capaccioli et al. 2015). NGC 4472 presents extended shells and fans of diffuse material, and Capaccioli et al. (2015) also detected an intra cluster light (ICL) contribution to the surface brightness profile. Irwin \& Sarazin (1996) found that the X-ray 
contours of NGC 4472 are elongated in the northeast-southwest direction, probably as a result of motion towards the centre of the Virgo cluster.

\subsection{NGC 5044}

NGC 5044 is the central and brightest galaxy of a rich and X-ray bright group (Mendel et al. 2008). Dust patches near the centre of the galaxy have been detected by Colbert et al. (2001). X-ray observations of NGC 5044 show two cavities and cooler filamentary X-ray emission, which are thought to have been caused by a dynamical perturbation induced by the accretion of a low-mass satellite (Buote et al. 2003; Gastaldello et al. 2009).

\subsection{NGC 5846}

NGC 5846 is the optically most luminous galaxy in the centre of its group. Hubble Space Telescope (HST) images show the presence of a "parabolic" dust lane around the nucleus of the galaxy. X-ray observations (Trinchieri \& Goudfrooij 2002) show a disturbed hot gas morphology on arcsecond scales. Haynes \& Giovanelli (1991) found that the three-dimensional distribution of galaxies around NGC 5846 is a prolate cloud that is pointing towards the Virgo cluster.

\section{The Vegas Survey: observation and data reduction}

The VEGAS survey and the data reduction procedure adopted in this work are described by Capaccioli et al. (2015). The only significant change we make here concerns the sky background subtraction for objects with large angular extent (i.e. those filling the VST field of view). In Paper I we used a direct polynomial interpolation of regions surrounding our target galaxies that were identified as "background" after masking of bright sources. This procedure, according to our experience, works very well to isolate a statistically significant area for the background interpolation when the target has a angular extent that is much smaller than the VST field. However, experience gained in reducing images of NGC 4472 highlighted the need for a different approach, which we develop here. This modified approach involves both observations and data reduction.

We decided to adopt a step-dither observing strategy for galaxies with large angular extent, consisting of a cycle of short exposures centred on the target and on offset fields $(\Delta= \pm 1$ degree). With such a technique the background can be estimated from exposures taken as close as possible, in space and time, to the scientific images. This ensures better accuracy, reducing the uncertainties at very faint surface brightness levels, as found by Ferrarese et al. (2012). This observing strategy allowed us to build an average sky background of the night, which was subtracted from each science image.

The main advantage of the step-dither technique is the possibility of obtaining a larger field around galaxies with larger angular sizes. This reduces uncertainties in distinguishing pixels belonging to the target galaxy from the background, particularly in the outskirts of the target. On the other hand, with this technique galaxy and background exposures are not simultaneous and more telescope time is consumed with respect to the standard dithering strategy.

In the subsample of VEGAS analyzed in this work, the images of NGC 4472 and NGC 4365 were acquired with standard dithering strategy, while for NGC 1399, NGC 3923, NGC 5044, and NGC5846 we adopted the step-dither strategy.
Table 2. VST exposures used in this work.

\begin{tabular}{lccc}
\hline \hline Object & Band & $\begin{array}{c}T_{\exp } \\
{[\mathrm{s}]}\end{array}$ & $\begin{array}{c}F W H M^{a} \\
\text { [arcsec] }\end{array}$ \\
\hline NGC 3923 & $g$ & 9600 & 1.08 \\
& $i$ & 12450 & 0.70 \\
NGC 4365 & $g$ & 5475 & 0.86 \\
& $i$ & 6125 & 0.73 \\
NGC 5044 & $g$ & 14100 & 1.13 \\
& $i$ & 4800 & 1.06 \\
NGC 5846 & $g$ & 8550 & 0.96 \\
& $i$ & 1650 & 0.90 \\
\hline
\end{tabular}

Notes. ${ }^{(a)}$ Median value of the FWHM.

The data used in this paper consist of exposures in $g$ and $i$ SDSS bands (Table 2) obtained with VST + OmegaCAM, both in service and visitor mode with the following constraints:

- $S / N \geq 3$ per $\operatorname{arcsec}^{2}$;

- dark time;

- seeing $\sim 1^{\prime \prime}$;

- airmass $\leq 1.2$.

By assuming the above values for the total integration time, the ETC for OmegaCam (version 6.2.0) ensures that we should reach a limiting magnitude in the $B$ band of $\mu=29 \mathrm{mag} / \mathrm{arcsec}^{2}$ $\left(\mu_{g} \sim 28.6 \mathrm{mag} / \operatorname{arcsec}^{2}\right.$ in the $\mathrm{g}$ band) with a $S / N \sim 3$ per $\operatorname{arcsec}^{2}$. The fainter magnitude levels of the azimuthally averaged surface brightness profiles are due to the increasing collecting area with the galactocentric radius.

All the data were processed with the VST-Tube pipeline (Grado et al. 2012). Details about the various steps of the data reduction are described in Appendix A of Capaccioli et al. (2015). The sky background subtraction procedure for data taken with the standard strategy is illustrated in Capaccioli et al. (2015).

For images taken with the step-dither technique, the only difference in our analysis concerns the estimation of the background. In these cases, an average sky image, relative to each night, was derived from the exposures of the offset fields, in which all bright sources were masked. The VST mosaics of galaxies in this work, showing the masking of bright sources in the field, are in Appendix A. This mean sky frame was then scaled to take into account transparency variations and subtracted from the science frame (see Iodice et al. 2016, for details). In this paper, all magnitudes are quoted in the AB system.

\section{Data analysis}

\subsection{Isophotal analysis}

The most critical operation in deep photometric analysis is the estimation and subtraction of the sky background. In Paper I we discuss pros and cons of a background subtraction made with the direct polynomial interpolation and by using a step-dither technique. After the background subtraction, described in Sect. 3, we applied the methodology described by Pohlen \& Trujillo (2006) to quantify the sky variations and subtract any possible residuals. Following this procedure, on the sky-subtracted mosaics in both the $g$ and $i$ bands, we masked all the bright sources and used the IRAF ${ }^{3}$ task ELLIPSE to extract azimuthally averaged

\footnotetext{
3 IRAF (Image Reduction and Analysis Facility) is distributed by the National Optical Astronomy Observatories, which is operated by the Associated Universities for Research in Astronomy, Inc. under cooperative agreement with the National Science Foundation.
} 
intensity profiles in elliptical annuli out to the edges of the frame by fixing both the ellipticity $(\epsilon)$ and the position angle (PA) to the values best describing the isophotes at the semi-major axis $R \sim 1$ arcmin. By extrapolating the trend in the outer regions of these intensity profiles for each galaxy in our sample, we found that the error on the average value of the residual background is $0.1 \leq \sigma_{g} \leq 0.2$ counts in the $g$ band and $0.2 \leq \sigma_{i} \leq 0.5$ counts in the $i$ band. These fluctuations of the sky background have been taken into account in the error estimates we quote on our surface brightness measurements. The VST $g$ band images of NGC 1399, NGC 3923, NGC 4365, NGC 4472, NGC 5044, and NGC 5846, coloured by surface brightness, are shown in the top panels of Figs. 1, D.1-D.5, respectively.

In the bottom left panels of the same figures we show the ellipticity $(\epsilon)$ and position angle (PA) profiles resulting from our isophotal analysis, performed via the ELLIPSE task, leaving ellipticity and position angle free and sampling the semi-major axis with a variable bin (as described in Paper I). ELLIPSE computes the intensity, $I(a, \theta)$, azimuthally sampled along an elliptical path described by an initial guess for the isophote centre, $(X, Y)$, ellipticity, $\epsilon$, and semi-major axis position angle, $\theta$, at different semi-major axis lengths, $a$. At a given $a_{0}, I\left(a_{0}, \theta\right)$ is expanded into a Fourier series as

$I\left(a_{0}, \theta\right)=I_{0}+\sum_{k}\left(a_{k} \sin (k \theta)+b_{k} \cos (k \theta)\right)$

according to Jedrzejewski (1987). The best-fit parameters are those minimizing the residuals between the actual and the model isophotes; $a_{k}$ and $b_{k}$ are the coefficients measuring the deviations from a pure ellipse, including the signature of boxiness and/or diskiness (Bender et al. 1989). An image sampling is performed at each iteration of the isophote fitting. Three methods are available for sampling an image along the elliptical path: bilinear interpolation and either mean or median over elliptical annulus sectors. Integration over elliptical annulii is preferred to extract all the information from an image and to avoid the possibility that some pixels in the image might never be sampled. For this reason, the SB profiles in this work have been extracted by performing a median over elliptical annuli, and by applying a $\mathrm{k}$-sigma clipping algorithm for cleaning deviant sample points at each annulus. The combined use of the median and sigma clipping algorithm improves the isophotal fitting, ensuring the rejection of not entirely masked features such as stars, defects, and outskirts of neighbouring galaxies.

For all the galaxies we find an increase of the ellipticity in the outer regions, indicating the presence of a more flattened outer component with a significant gradient in the PA. We return to this point in Sect. 5 .

In the bottom right panels we show $g$ - and $i$-band VST surface brightness profiles, including comparisons with other measurements in the literature. No correction for seeing blurring is applied to the inner regions of the profiles. The offsets relative to the VST $g$ band providing the best match to our photometry are +0.7 mag for the $V$-band profile of NGC 3923 by Sikkema et al. (2007), +0.33 mag for the $V$-band profile of NGC 4472 by Kormendy et al. (2009), $+0.2 \mathrm{mag}$ for the $V$-band profile of NGC 4365 by Kormendy et al. (2009), and +0.23 mag for the $V$ band profile of NGC 5846 by Kronawitter et al. (2000). Outside the seeing-blurred cores, despite the different colour bands, the agreement with the literature is good. The reliability of the surface brightness measurements in the faint outskirts of the galaxies has been tested by extracting the surface brightness profiles in azimuthal sectors (see Appendix B for details). The errors associated with our surface brightness measurements were computed as in Paper I, and take into account both the uncertainties on the photometric calibration and on sky subtraction. All the profiles are very extended and deep $\left(29 \leq \mu_{g} \leq 30.5 \mathrm{mag} / \operatorname{arcsec}^{2}\right)$ and the azimuthally averaged surface brightness of the four objects spans almost one dex.

As in Paper I, we use the luminosity profiles of the galaxies, together with their ellipticity profiles, to compute the growth curves, which we extrapolate to the derive total magnitudes $m_{\mathrm{T}}$, effective radii $R_{\mathrm{e}}$ and corresponding effective magnitude $\mu_{\mathrm{e}}$ (Table 3).

In Fig. 2, we plot all the profiles as a function of galactocentric radius normalized to the effective radius $R / R_{\mathrm{e}}$. This comparison shows that, in our sample, we have two kinds of profiles: those with an "excess" of light in the outer regions, which tend to flatten in the outer regions, and those for which the surface brightness falls off more rapidly. The differences in the shape of the light profiles may be signatures of different dynamical states for the stellar halos surrounding these galaxies; we discuss this point in Sect. 7.

The mean $(g-i)$ colour profiles for galaxies in our sample are shown in right panel of Fig. 2. The $(g-i)$ colour profile for NGC 1399 is from Iodice et al. (2016), while that for NGC 4472 is from Capaccioli et al. (2015). The colours of all galaxies are redder than those those typically found for ETGs. As shown by Ann (2010), galaxies that are beyond the red edge of the red sequence can have different origins; many of them have dust lanes or are merging galaxies, and the dust obscuration is mainly responsible for their red colours. Consistent with this picture, all the galaxies in our sample show the presence of dust lanes, shells, and other signs of ongoing interactions. All the colour profiles display in the central regions $\left(0.01<R / R_{\mathrm{e}}<0.1\right)$, where on average the galaxies are redder, small but significant negative gradients typical of early types (La Barbera et al. 2010; Tortora et al. 2010). At a different radius for each galaxy, the colours develop a steeper gradient, becoming bluer (for NGC 1399, NGC 4365, NGC 5044, and NGC 5846) or redder (NGC3923 and NGC 4472). In Sect. 5 we show that the discontinuities observed in the colour profiles correspond to the transition radii observed in each surface brightness profile, which are defined as the locations of the transition between two different fit components.

\subsection{Correction for the PSF}

It is well known (Capaccioli \& de Vaucouleurs 1983) that the faint outskirts of galaxies can be contaminated by light scattered from the bright core by the telescope and the atmosphere. In order to account for the artificial halos created by scattered light, the point spread function (PSF) must be mapped out to a radial distance that is at least comparable to the extent of the galaxian halo. An extended PSF for VST was derived in Appendix B of Paper I. There we also show how to estimate the amount of scattered light by deconvolving a noiseless model of each galaxy with the IRAF task LUCY, which is based on the Lucy-Richardson algorithm (Lucy 1974; Richardson 1972).

As discussed in Paper I, the effect of the scattered light is very different at the same surface brightness level between galaxies with large and small angular extent. We found that the surface brightness profiles of smaller sources in the field of NGC 4472 were marginally affected $(\sim 0.2 \mathrm{mag})$ by the extended PSF at $\mu_{g} \sim 29 \mathrm{mag} / \operatorname{arcsec}^{2}$. Galaxies with a large angular extent, however, were not significantly affected by the PSF.

We also performed a plain numerical convolution for a set of azimuthally averaged light profiles for artificial galaxy models 

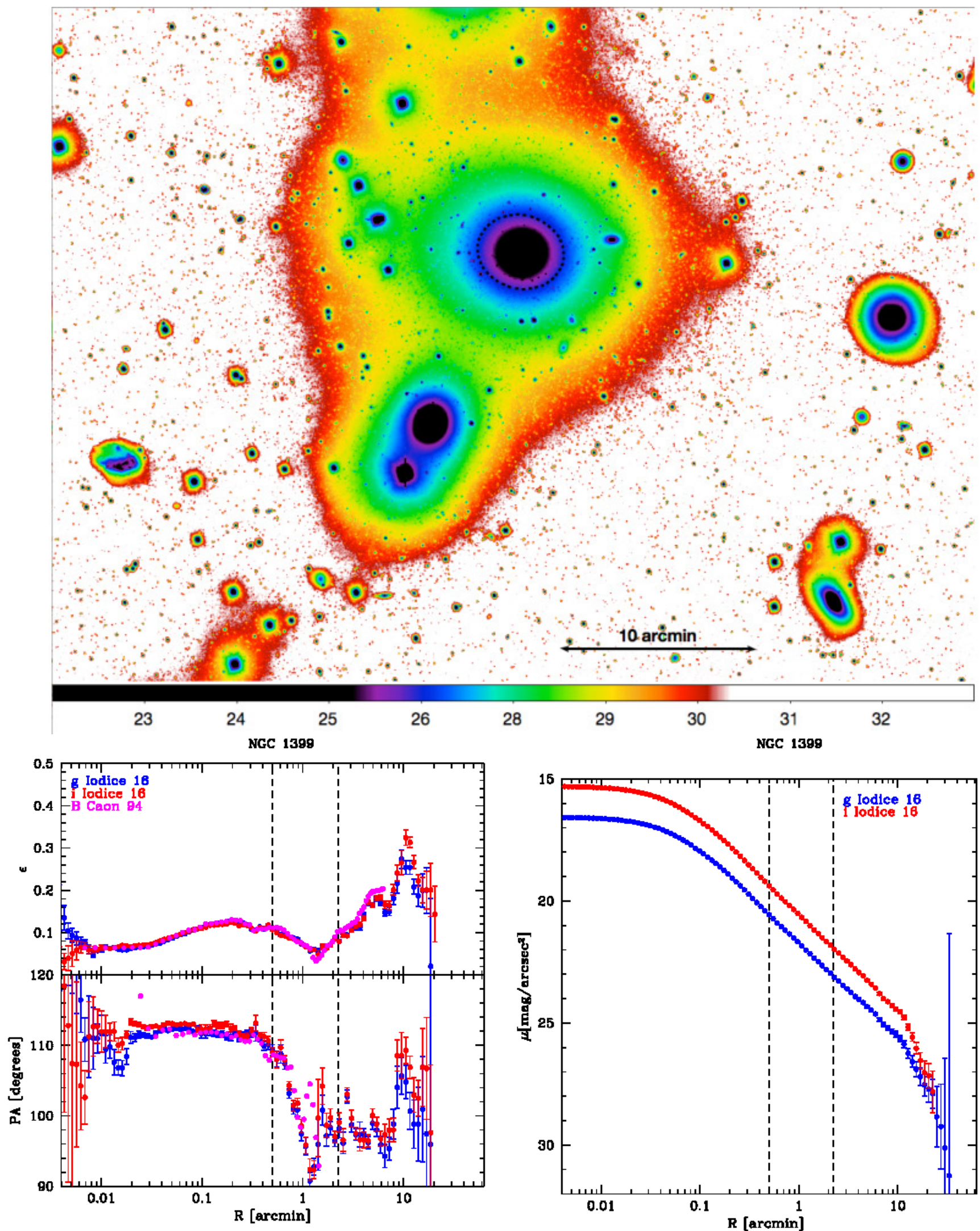

Fig. 1. Top: VST $g$-band sky-subtracted image of the field around NGC 1399. The colour scale represents surface brightness in mag/arcsec ${ }^{2}$. The dashed black contour indciates the transition radius in the surface brightness profile, defined in Sect. 5. North is up, east is to the left. Bottom left: ellipticity $(\epsilon)$ and position angle (PA) profiles for NGC 1399, in the $g$ band (blue points) and $i$ band (red points). Bottom right: azimuthally averaged surface brightness profiles in the $g$ (blue) and $i$ (red) bands. The dashed lines indicate the transition radii in the surface brightness profile, listed in Table 6, which represents the location of the transition between two fit conponents, as described in Sect. 5. 
Table 3. Distances and photometric parameters for the sample galaxies.

\begin{tabular}{|c|c|c|c|c|c|c|c|c|c|c|c|c|c|c|c|}
\hline Object & $\begin{array}{c}D \\
{[\mathrm{Mpc}]} \\
\text { (a) }\end{array}$ & $\begin{array}{c}A_{g} \\
{[\mathrm{mag}]} \\
\text { (b) }\end{array}$ & $\begin{array}{c}A_{i} \\
{[\mathrm{mag}]} \\
\text { (b) }\end{array}$ & $\begin{array}{c}\mu_{\mathrm{e}, g} \\
{\left[\mathrm{mag} / \operatorname{arcsec}^{2}\right]} \\
\text { (c) }\end{array}$ & $\begin{array}{c}r_{\mathrm{e}, g} \\
{[\operatorname{arcmin}]}\end{array}$ & $\begin{array}{c}\left(R_{\mathrm{e}, \mathrm{g}}\right)_{\text {maj }} \\
{[\mathrm{kpc}]}\end{array}$ & $\begin{array}{c}m_{\mathrm{T}, g} \\
{[\mathrm{mag}]} \\
(\mathrm{c})\end{array}$ & $\begin{array}{c}M_{\mathrm{T}, g} \\
{[\mathrm{mag}]} \\
(\mathrm{c})\end{array}$ & $\begin{array}{c}\mu_{\mathrm{e}, i} \\
{\left[\mathrm{mag} / \operatorname{arcsec}^{2}\right]} \\
\text { (c) }\end{array}$ & $\begin{array}{c}r_{\mathrm{e}, i} \\
{[\operatorname{arcmin}]}\end{array}$ & $\begin{array}{c}\left(R_{\mathrm{e}, \mathrm{i}}\right)_{\mathrm{maj}} \\
{[\mathrm{kpc}]}\end{array}$ & $\begin{array}{c}m_{\mathrm{T}, i} \\
{[\mathrm{mag}]} \\
(\mathrm{c})\end{array}$ & $\begin{array}{c}M_{\mathrm{T}, i} \\
{[\mathrm{mag}]} \\
(\mathrm{c})\end{array}$ & $\begin{array}{l}\left\langle\epsilon_{\text {in }}\right\rangle \\
\text { (d) }\end{array}$ & $\begin{array}{r}\left\langle\epsilon_{\text {out }}\right\rangle \\
\text { (d) }\end{array}$ \\
\hline & 9 & 05 & 0.04 & 24.55 & 588 & & 55 & -2305 & - & 5 & & 7.51 & -24.09 & 0 & 0.203 \\
\hline & & & & & & & & & & & & & & & 2 \\
\hline & & 0.08 & & & & & & & & & & 8.52 & & & 0.282 \\
\hline & & 0.08 & & & & 29 & 8.37 & -22.77 & & 4. & 3 & 6.92 & & & 0.205 \\
\hline & .2 & 0.26 & & & & & 9. & -2 & & 3. & & 8.76 & & 68 & 0.340 \\
\hline NGC 5846 & 24.9 & 0.21 & 0.11 & 24.72 & 4.62 & 33.41 & 9.21 & -22.77 & 23.32 & 3.70 & 26.80 & 7.99 & -24.00 & 0.067 & 0.167 \\
\hline
\end{tabular}

Notes. ${ }^{(a)}$ Distances of NGC 3923, NGC 5044, and NGC 5846 are from Tonry et al. (2001); distances of NGC 4365 and NGC 1399 are from Blakeslee et al. (2009); and the distance of NGC 4472 is from Mei et al. (2007). ${ }^{(b)}$ Sources for the extinction correction in the $g$ and $i$ band are Schlegel et al. (1998) for all objects but NGC 4472 for which we adopt the value used in Paper I. ${ }^{(c)}$ Derived by integrating the growth curves and corrected for interstellar extinction. ${ }^{(d)} g$ band mean ellipticity in the ranges $-1.5<\log \left(R / R_{\mathrm{e}}\right)_{\text {maj }}<-0.5\left(\epsilon_{\mathrm{in}}\right)$ and $0<\log \left(R / R_{\mathrm{e}}\right)_{\text {maj }}<0.5\left(\epsilon_{\mathrm{out}}\right)$.
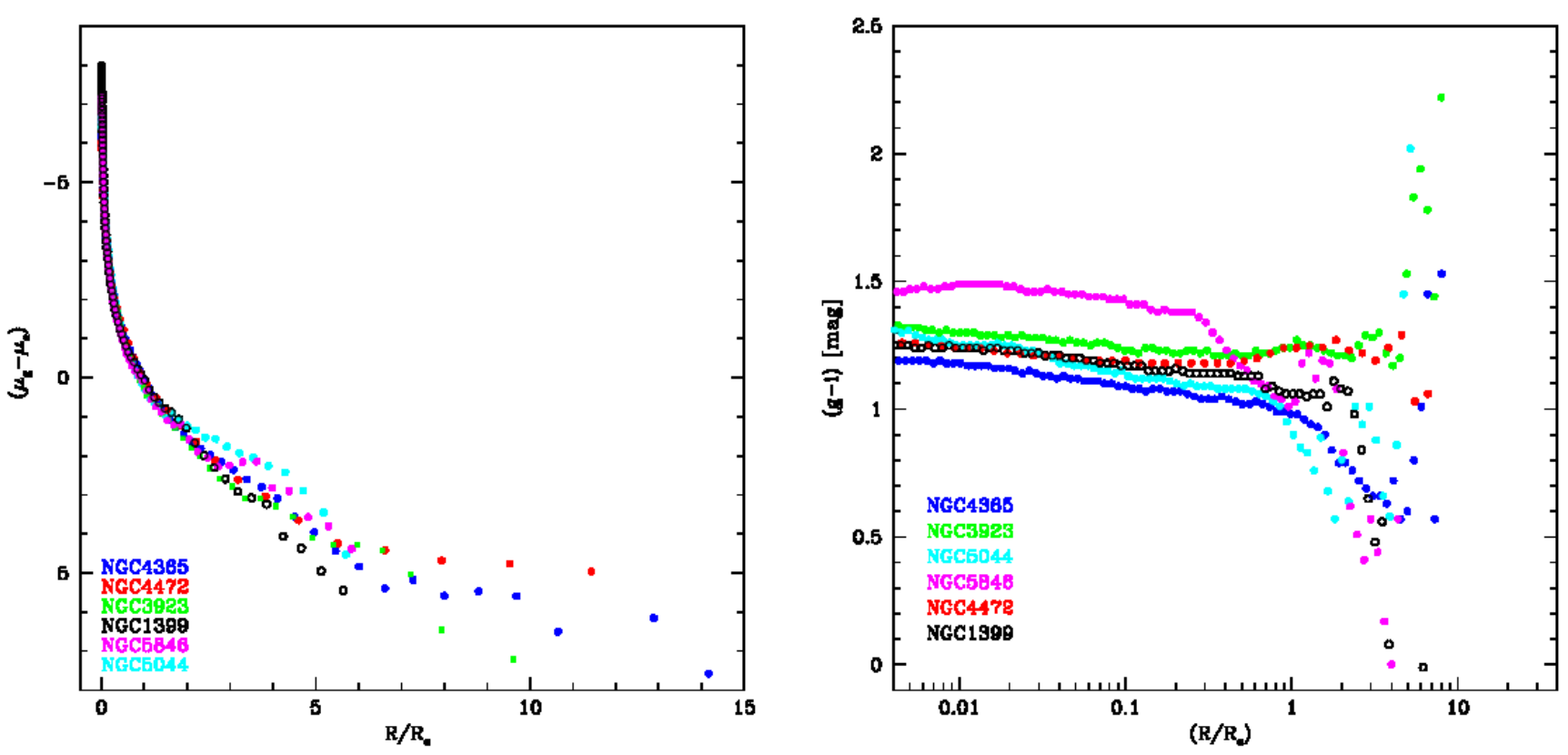

Fig. 2. Left: azimuthally averaged surface brightness profiles in the $g$ band scaled to their effective magnitude, as a function of galactocentric radius normalized to the effective radius, $R / R_{\mathrm{e}}$. The innermost region is not corrected for seeing convolution (typical $F W H M=0.8$ arcsec). Right: $(g-i)$ colour profiles of the galaxies in our study.

with different sizes. The difference between the model and its convolution provides an estimate of the effect of the extended PSF, at different surface brightness levels, on galaxies with different sizes. Details and results of these numerical experiments will be presented in a forthcoming paper (Spavone et al., in prep.). We conclude that no significant contribution of scattered light to the azimuthally averaged light profile is present in the faintest, outermost regions of the six giant galaxies we study in this paper.

\section{Fitting ellipticity and light distribution}

\subsection{Deprojected flattening}

It is not easy to make a meaningful comparison between the apparent ellipticity curves of different ETGs when their intrinsic shapes and inclinations, $\theta$, are unknown. As a first-order approximation to the average trend in our sample we have deprojected the observed flattening profiles by adopting the classical prescription (Binney \& Merrifield 1998),

$\xi^{2} \sin ^{2} \theta+\cos ^{2} \theta=\left\{\begin{aligned} q^{2} & \text { (oblate) } \\ 1 / q^{2} & \text { (prolate) }\end{aligned}\right.$

where $q$ and $\xi$ are the apparent and true axial ratios, respectively. We have assumed that the dominant spheroidal components of all our six galaxies are oblate and have $\langle\xi\rangle=0.62$ (Binney \& de Vaucouleurs 1981).

Results are shown in Fig. 3, including ellipticity profiles for NGC 1399 and NGC 4472. The solid line represents a running mean of the complete dataset. Individual profiles show a wide dispersion, but an overall increase of flattening from 0.38 to 0.5 is apparent over a range $0.01<R / R_{\mathrm{e}}<6$ along the major axis. This is broadly consistent with previous findings. For example Gonzalez et al. (2005) found that a sample of BCGs to be well described by a two-component model, in which the outer component was more elongated than the inner component that they associated with the BCG. 


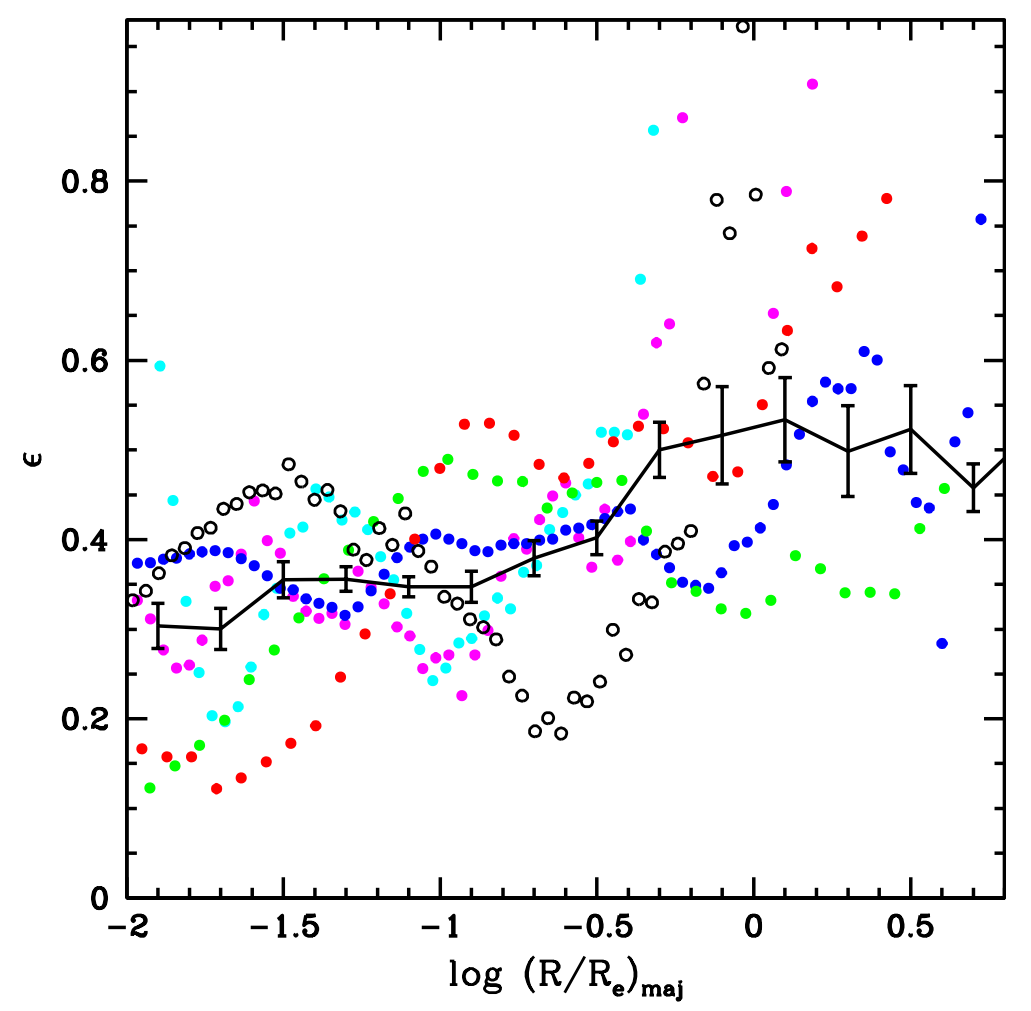

Fig. 3. Deprojected ellipticity $(\epsilon)$ profiles in the $g$ band, as a function of $\log \left(R / R_{\mathrm{e}}\right)_{\text {maj }}$. The solid black line is the mean trend of the whole sample. The error bars trace the dispersion of the points. The colour code is the same as that in Fig. 2.

\subsection{Fitting the light distribution with two-component models}

The remainder of our analysis focusses on the projected onedimensional (ellipsoidally averaged) surface brightness profiles of our sample galaxies. As noted in the introduction, there is considerable evidence in the literature that the light profiles of many of the most massive ETGs are not well fitted by a single Sérsic law and at least one additional component is needed (Seigar et al. 2007; Donzelli et al. 2011; Arnaboldi et al. 2012; Iodice et al. 2016). This second component is sometimes (but not universally) interpreted as evidence for a stellar halo or "intracluster light".

Theoretical models suggest that massive ETGs accumulate the bulk of their stellar mass by accretion. For this reason, the "stellar halo" in these galaxies should be identified with the component dominating the stellar mass. From an observational point of view, it is not straightforward to separate the in situ and the accreted component in ETGs, since they have similar physical properties and are well mixed together. The overall profile is comprised of different contributions and for this reason theory suggests that the surface brightness profile of ETGs should be described by the superposition of different components.

Since we are interested in the study of the stellar distribution in the outer envelopes of our sample galaxies, we do not use the $\chi^{2}$ statistics in our fitting procedure. The data points corresponding to the central regions of the galaxies, in fact, with their small uncertainties have considerable weight in determining the best-fit solution obtained by minimizing the $\chi^{2}$, while the outer regions with bigger errors have no weight. This approach could produce erroneous results when the functional form of the model is not known a priori, and an empirical model has to be assumed to reproduce the light distribution. To avoid this problem, we adopt the same approach described by Seigar et al. (2007) and performed least-square fits using a Levenberg-Marquardt algorithm, in which the function to be minimized is the rms scatter, defined as $\Delta=\sqrt{\frac{\sum_{i=1}^{m} \delta_{i}^{2}}{m}}$, where $m$ is the number of data points and $\delta_{i}$ is the $i$ th residual. In all the fit presented below, the innermost seeing-dominated regions $(\sim 1.5 \times F W H M)$, indicated with dashed lines, were excluded.

Although we average azimuthally in elliptical annulli, this does not mean that the components fit to the resulting radial profile should be necessarily expected to be elliptical structures or even azimuthally smooth. This is simply an empirical approach to quantify the amount of stellar material as a function of galaxy radius.

As shown in Sect. 4, we find two apparent types of surface brightness profiles in our sample: those with steeper slopes in the outer regions and those with shallower slopes. In either case, the single Sersic $R^{1 / n}$ model provides a poor description of the shape of the profile. This can be clearly seen from the residuals with respect to a single Sérsic fit shown in Fig. D.6.

For this reason, we first present models of the surface brightness profiles of galaxies in our sample with a double Sérsic law (Sérsic 1963; Caon et al. 1993),

$\mu(R)=\mu_{\mathrm{e}}+k(n)\left[\left(\frac{R}{r_{\mathrm{e}}}\right)^{1 / n}-1\right]$,

where $k(n)=2.17 n-0.355, R$ is the galactocentric radius, and $r_{\mathrm{e}}$ and $\mu_{\mathrm{e}}$ are the effective radius and surface brightness. This asymptotic expansion provides an accurate fit for $n \geq$ 0.36 (Ciotti \& Bertin 1999; MacArthur et al. 2003). We found that this model converges to a best-fit solution with a physically meaningful value for only two galaxies, NGC 5044 and NGC 5846. In these cases the fit yields very small Sérsic indices ( $n_{2} \sim 0.7$ and $n_{2} \sim 0.4$, respectively) for the outer components.

For the cases in which our double-Sérsic fit did not converge, we imposed an exponential profile $(n=1)$ on the outer 
Table 4. Best-fitting structural parameters for a two-component fit.

\begin{tabular}{|c|c|c|c|c|c|c|c|c|c|c|c|c|c|}
\hline Object & $\begin{array}{c}\mu_{\mathrm{e} 1} \\
{\left[\mathrm{mag} / \operatorname{arcsec}^{2}\right]}\end{array}$ & $\begin{array}{c}r_{\mathrm{e} 1} \\
{[\operatorname{arcsec}]}\end{array}$ & $n_{1}$ & $\begin{array}{c}\mu_{\mathrm{e} 2} \\
{\left[\mathrm{mag} / \operatorname{arcsec}^{2}\right]}\end{array}$ & $\begin{array}{c}r_{\mathrm{e} 2} \\
{[\operatorname{arcsec}]}\end{array}$ & $n_{2}$ & $\begin{array}{c}\mu_{0} \\
{\left[\mathrm{mag} / \operatorname{arcsec}^{2}\right]}\end{array}$ & $\begin{array}{c}r_{h} \\
{[\operatorname{arcsec}]}\end{array}$ & $\begin{array}{c}m_{\mathrm{T}, b} \\
{[\mathrm{mag}]}\end{array}$ & $\begin{array}{c}m_{\mathrm{T}, h} \\
{[\mathrm{mag}]}\end{array}$ & $f_{h}$ & $\begin{array}{c}R_{\mathrm{tr}} \\
{[\operatorname{arcmin}]}\end{array}$ & $\begin{array}{c}\mu_{\mathrm{tr}} \\
{\left[\mathrm{mag} / \mathrm{arcsec}^{2}\right]}\end{array}$ \\
\hline NGC 1399 & $21.3 \pm 0.2$ & $45 \pm 4$ & $3.9 \pm 0.3$ & - & - & - & $23.4 \pm 0.1$ & $292 \pm 4$ & 9.67 & 9.08 & $64 \%$ & $2.2 \pm 0.3$ & 24.0 \\
\hline NGC 3923 & $22.2 \pm 0.3$ & $77 \pm 11$ & $3.8 \pm 0.3$ & - & - & - & $24.4 \pm 0.2$ & $273 \pm 13$ & 9.43 & 10.21 & $32 \%$ & 2.2 & 25.3 \\
\hline NGC 4365 & $21.9 \pm 0.2$ & $60 \pm 7$ & $4.1 \pm 0.2$ & - & - & - & $23.4 \pm 0.1$ & $166 \pm 4$ & 9.66 & 10.26 & $36 \%$ & $2.9 \pm 0.8$ & 24.8 \\
\hline NGC 4472 & $22.5 \pm 0.2$ & $148 \pm 14$ & $4.3 \pm 0.2$ & - & - & - & $26.9 \pm 0.6$ & $1286 \pm 530$ & 8.29 & 9.36 & $27 \%$ & $18.2 \pm 8.9$ & 27.8 \\
\hline NGC 5044 & $22.9 \pm 0.1$ & $62 \pm 4$ & $3.3 \pm 0.1$ & $27.00 \pm 0.09$ & $685 \pm 33$ & $0.7 \pm 0.1$ & - & - & 9.43 & 10.60 & $40 \%$ & $3.6 \pm 0.3$ & 26.2 \\
\hline NGC 5846 & $24.2 \pm 0.3$ & $167 \pm 25$ & $5.3 \pm 0.3$ & $27.22 \pm 0.12$ & $820 \pm 17$ & $0.40 \pm 0.07$ & - & - & 9.72 & 9.26 & $60 \%$ & $8.7 \pm 2.0$ & 27.0 \\
\hline
\end{tabular}

Notes. Columns 2-4 report effective magnitude, effective radius, and Sérsic index for the inner component of each fit. Columns 5-7 list the same parameters for galaxies we fit with an outer Sérsic component, whereas Cols. 8 and 9 list the central surface brightness and scale length for galaxies we fit with an outer exponential component. Columns 10 and 11 report the total magnitude of the inner Sérsic $\left(m_{\mathrm{T}, b}\right)$ and outer exponential or Sérsic component $\left(m_{\mathrm{T}, h}\right)$. Columns 12-14 give the relative fraction of the outer component with respect to the luminosity of the galaxy, transition radius, and surface brightness at that radius, respectively.

component, given by the equation

$\mu(R)=\mu_{0}+1.086 \times R / r_{h}$,

where $\mu_{0}$ and $r_{h}$ are the central surface brightness and exponential scale length, respectively. In these fits a Sérsic component without restrictions was retained for the inner regions.

These double Sérsic/Sérsic plus exponential fits were performed on the $g$-band azimuthally averaged light profile, excluding the core of the galaxies $(R \sim 2$ arcsec). The result of these fits and their residuals are shown in Fig. D.7, in which the core of each galaxies is indicated with a dashed line. In Table 4 we report the structural parameters of the best fit for each galaxy with the relative $95 \%$ confidence intervals. In Fig. D.8 we plot the results shown in Fig. D.7, but on a linear scale, as a function of $R / R_{\mathrm{e}}$. In this figure the distinction between the two types of surface brightness profiles is more clear. Specifically, we can distinguish the profiles of NGC 1399, NGC 5044, and NGC 5846, which show a downward inflection, from those of NGC 3923, NGC 4365, and NGC 4472, which have an upward inflection instead. According to our multicomponent modelling, all the upward inflections are the result of a transition from a high- $n$ inner component to a larger, lower- $n$, outer component. Downward inflections are, instead, the natural cut-off of a low- $n$ component. This implies that a downward inflection should be accompanied by an upward inflection at some smaller radius.

We found that the inner components of each fit have effective radii $r_{\mathrm{e}} \sim 5-25 \mathrm{kpc}$ (45-202 arcsec), with an average value of $r_{\mathrm{e}} \sim 12 \mathrm{kpc}$, and Sérsic indices $n \sim 3-6$, with an average value of $n \sim 4.3$. These values are consistent with those reported by Gonzalez et al. (2003) and Donzelli et al. (2011), who for their samples of BCGs found $r_{\mathrm{e}} \sim 5-15 \mathrm{kpc}$ and $n \sim 4.4$.

We used these fits to derive the total magnitude of the inner Sérsic component, $m_{\mathrm{T}, b}$, and outer (exponential or Sérsic) component, $m_{\mathrm{T}, h}$, and the relative contribution of the outer halo with respect to the total galaxy light, $f_{h}$, reported in Table 4 .

From this analysis we have identified a radius for each galaxy in our sample that marks the transition between the inner and outer components of our two-component fit. We label this empirically defined "transition radius" $R_{\mathrm{tr}}$. This transition occurs at very faint levels of surface brightness $\left(\mu_{\mathrm{tr}}\right)$ for all our galaxies. As mentioned in Sect. 4, a discontinuity in the ellipticity, PA, and $(g-i)$ colour profiles of all six galaxies occurs around $R_{\mathrm{tr}}$.

For NGC 1399 and NGC 5846 the outer halo component becomes even brighter than the inner component at $R \geq R_{\mathrm{tr}}$, where the relative contribution of the outer halo with respect to the total galaxy light $\left(f_{h}\right)$ reaches $60 \%$ and $55 \%$, respectively. For all the other galaxies the inner Sérsic component dominates the
Table 5. Correlation between outer profiles and environment.

\begin{tabular}{lcc}
\hline \hline Object & $N_{\text {gal }}$ & slope \\
\hline NGC 1399 & 54 & $13.13 \pm 1.51$ \\
NGC 3923 & 3 & $5.64 \pm 0.85$ \\
NGC 4365 & 51 & $5.82 \pm 2.08$ \\
NGC 4472 & 39 & $2.26 \pm 0.30$ \\
NGC 5044 & 127 & $16.65 \pm 2.53$ \\
NGC 5846 & 85 & $7.10 \pm 3.45$ \\
\hline
\end{tabular}

Notes. Column 2 reports the number of physically related galaxies (the spread of velocities for the individual galaxies is about $150 \mathrm{~km} \mathrm{~s}^{-1}$ ) in a field of 1 square degree around each galaxy in our sample. Column 3 is the slope of the outer halo surface brightness profiles, obtained by performing a power-law fit in the region of the outer envelope derived from the two-component model, that is between $R_{\text {tr }}$ and the last measured point.

light distribution, but the outer halo component is never negligible with relative fractions between $28 \%$ and $39 \%$. Since there is no clear reason to believe that in massive elliptical galaxies the outer component in a fit such as this accounts for most of the accreted mass, the halo mass fractions reported in Table 4 should be considered a lower limit for the total accreted mass. We return to this point in the following section.

In order to investigate the possibility of a correlation between environment and the shape of surface brightness profiles, in Fig. 4 we plot the slope of the outer halo surface brightness profiles (Fig. D.7) against the number of physically related galaxies in a field of 1 square degree around each galaxy; the spread of velocities for the individual galaxies is about $150 \mathrm{~km} \mathrm{~s}^{-1}$. The profile slope was obtained by performing a power-law fit in the region of the outer envelope derived from the two-component model, that is between $R_{\mathrm{tr}}$ and the last measured point (see Table 5). This figure hints at a trend such that galaxies in more dense environments have halo light profiles that are steeper than those in less dense fields. In order to evaluate the robustness of the correlation between the slopes and the density of the environment in Fig. 4, we estimated the Spearman rank correlation coefficient (SRCC; see Eq. 14.6.1 in Numerical Recipes), which ranges in the $[-1,1]$ interval and measures the degree of correlation between two variables. According to this test, two variables are positively/negatively correlated if the SRCC is significantly larger/smaller than zero for the two variables. As seen in Fig. 4, the main uncertainty on the correlation is given by the large error bars, hence we decided to use a Monte Carlo approach to the Spearman test to establish the significance of the correlation. We 


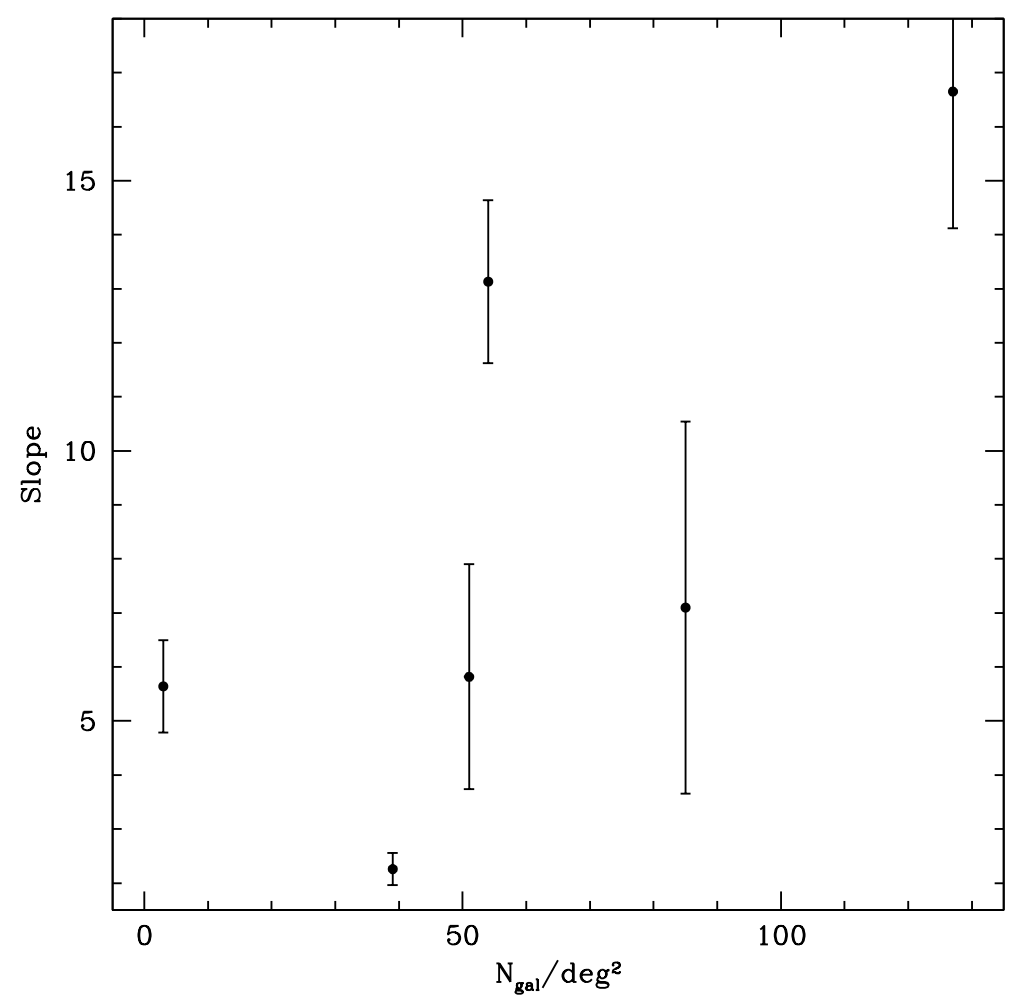

Fig. 4. Power-law slope of the outer halo surface brightness profiles (between $R_{\mathrm{tr}}$ and the last measured point), as a function of the number of physically related galaxies in a field of 1 square degree around each galaxy in our sample; the spread of velocities for the individual galaxies is about $150 \mathrm{~km} \mathrm{~s}^{-1}$.

ran 1000 experiments in which we perturbed the data assuming a normal distribution around each data point with the standard deviation given by the data error and computed the SRCC at every extraction. The mean SRCC we obtained after the 1000 random experiments is $0.67 \pm 0.20(1 \sigma)$. Even if we assume the more conservative standard error on SRCC, i.e. $0.6325 / \sqrt{N-1}=0.3$ for $N=6$, this test suggests that the slope and density of the environment are significantly correlated at least at $2 \sigma$ level. We believe that this is a new and interesting result to check on larger samples.

\subsection{Fitting the light distribution with three-component models}

Numerical simulations predict that stars accreted by BCGs account for most of the total galaxy stellar mass ( $90 \%$ on average), while in situ stars significantly contribute significantly to the surface brightness profile only out to $R \sim 10 \mathrm{kpc}$ (Cooper et al. 2013, 2015; Rodriguez-Gomez et al. 2016). The overall accreted profile is built up by contributions from several significant progenitors, which can be divided into two broad types. Stars from massive progenitors, which sink rapidly by dynamical friction, are centrally concentrated and have a shape resembling that of the overall galaxy profile, while less significant progenitors have larger effective radii and lower Sérsic indices (a detailed discussion of the dynamical principles is given by Amorisco 2017).

For this reason, theory suggests that the surface brightness profile of an ETG should be well described by the superposition of an inner Sérsic profile representing the (sub-dominant) in situ component in the central regions, another Sérsic profile representing the (dominant) superposition of the relaxed, phase-mixed accreted components, and an outer diffuse component representing unrelaxed accreted material (streams and other coherent concentrations of debris), which does not contribute any significant surface density to the brighter regions of the galaxy. (Cooper et al. 2015) found an $n \sim 1$ fitting component to be a reasonable empirical description of this unrelaxed debris in the azimuthal average. The stellar population of the in situ component is expected to be very similar to the dominant relaxed accreted component. As they are well mixed together, the sum of these two components is expected to have a smooth distribution with only faint features to suggest they are distinct.

Following these theoretical predictions, we described the surface brightness profiles of our six galaxies with a threecomponent model: a Sérsic profile for the centrally concentrated in situ stars, a second Sérsic for the relaxed accreted component, and an exponential or Sérsic component for the diffuse and unrelaxed outer envelope.

A three-component fit may suffer from substantial degeneracy between parameters. However, the numerical simulations cited above also predict that, for early-type galaxies in the mass range we are looking at, the variation of $n$ with halo mass is weak, especially for the in situ component. There is somewhat more variation with $n$ for the accreted stars, particularly with halo mass rather than stellar mass. Therefore, to mitigate the degeneracy in parameters and provide estimates of accreted components that are closely comparable to the results of numerical simulations, we fixed $n \sim 2$ for the in situ component of our three-component fits. We allowed small variations of \pm 0.5 around the mean value of $n=2$, since this would bracket the range of $n$ in the simulations and allows us to obtain a better fit. The Sérsic parameters for the accreted component were left free. We adopt this typical value of $n$ for the in situ component on the basis of the results of Cooper et al. (2013) for massive galaxies (see their Fig. 7 in).

The reason for the weak variation of $n$ with stellar mass and halo mass is because in situ stars are relatively deeply embedded in the oldest and most stable part of their host potential 
Table 6. Best-fitting structural parameters for a three-component fit in which the Sersic index of the inner component is fixed.

\begin{tabular}{|c|c|c|c|c|c|c|c|c|c|c|c|c|c|c|}
\hline bje & $\begin{array}{c}\mu_{\mathrm{e} 1} \\
{\left[\mathrm{mag} / \operatorname{arcsec}^{2}\right]}\end{array}$ & $\begin{array}{c}r_{\mathrm{e} 1} \\
\text { [arcsec] }\end{array}$ & $n_{1}$ & $\begin{array}{c}\mu_{\mathrm{e} 2} \\
{\left[\mathrm{mag} / \operatorname{arcsec}^{2}\right]}\end{array}$ & $\begin{array}{c}r_{\mathrm{e} 2} \\
{[\operatorname{arcsec}]}\end{array}$ & $n_{2}$ & $\begin{array}{c}\mu_{0} \\
{\left[\mathrm{mag} / \operatorname{arcsec}^{2}\right]}\end{array}$ & $\begin{array}{c}r_{h} \\
\text { [arcsec] }\end{array}$ & $\begin{array}{c}m_{\mathrm{T}, 1} \\
{[\mathrm{mag}]}\end{array}$ & & $\begin{array}{c}m_{\mathrm{T}, 3} \\
{[\mathrm{mag}]}\end{array}$ & $f_{h, \mathrm{~T}}$ & $\begin{array}{c}R_{\mathrm{tr} 1} \\
{[\operatorname{arcmin}]}\end{array}$ & $\begin{array}{c}R_{\mathrm{tr} 2} \\
{[\operatorname{arcmin}]}\end{array}$ \\
\hline & & & 2 & & & & & & & & & & & \\
\hline & & & 2 & & & & & & & & & & & \\
\hline & & & 2.2 & & & & & & & 9 & & & & \\
\hline & & & 2.5 & & & & & 00 & & & & & & \\
\hline & & & 2 & & & & & & & & 1 & & & \\
\hline & & + & 2 & $3.6 \pm 0.2$ & 110 & 2 & $5.0=$ & 29 & 11.4 & 0.0 & 9.7 & & & 4.2 \\
\hline
\end{tabular}

Notes. Columns 2-4 report effective magnitude and effective radius for the inner component of each fit. The Sérsic index for the in situ component was fixed to $n \sim 2$ using the models as a prior (Cooper et al. 2013). We allowed small variations of \pm 0.5 around the mean value of $n=2$. This would bracket the range of $n$ in the simulations and allows us to obtain a better fit. Columns 5-7 list the same parameters for the second component, whereas Cols. 8 and 9 list the central surface brightness and scale length for the outer exponential component. Columns 10-12 report the total magnitude of the inner Sérsic $\left(m_{\mathrm{T}, 1}\right)$ and outer components $\left(m_{\mathrm{T}, 2}\right.$ and $\left.m_{\mathrm{T}, 3}\right)$. Column 13 gives the total accreted mass fraction derived from our three-component fit, while Cols. 14 and 15 report the transition radii between two fit components.

and initially have quasi-exponential density profiles, i.e. they are late-type galaxies ${ }^{4}$. The high-energy (large apocentre) tails of these distributions then diffuse outward slightly in radius following relaxation in the near-equal mass ratio mergers that are responsible for making these massive galaxies early types by $z=0$ (e.g. Hilz et al. 2012). Only very massive mergers (in terms of total mass) affect the central regions of the potential in this way (hence the preservation of stable discs with exponential profiles, characteristic of ongoing dissipative star formation, as the dominant structural component of LTGs). Even in very massive clusters, most of the $\sim 10$ mergers that contribute significantly to the observed light profile are associated with dark halos of relatively low mass compared to the host (cluster) potential. Consequently, even though each progenitors has roughly similar stellar mass, most of these these mergers is (by the common convention) "minor", and the inner part of the potential with the in situ stars is rarely disturbed.

The results of these fits are shown in Fig. D.9 and the bestfitting parameters are reported in Table 6. As already explained in Sect. 5.2, even if we extract azimuthally averaged light profiles, the components fit to the radial profile are not necessarily elliptical structures. From this plot it appears that, as argued by Cooper et al. (2015), the radius $R_{\text {tr }}$ identified in Fig. D.7 marks the transition between different accreted components in different states of dynamical relaxation, rather than that between in situ and accreted stars. We found that the Sérsic indices for the accreted components in this case range between 1 and 2 . These values appear somewhat lower than those reported by (Cooper et al. 2013), but this is most likely because we included a third component that accounts for a significant fraction of the outermost regions of the profile. In support of this, in Appendix C, we report parameters obtained with this theoretically motivated approach without the outer exponential component, i.e. from a twocomponent Sérsic fit in which the inner component is fixed to $n \sim 2$. In that case the index of the outer component ranges $1.45<n_{2}<6.10$. We derived the total accreted mass fraction $f_{h, \mathrm{~T}}$ in Table 6 using the sum of both the "accreted" components in our three-component fit. As discussed further in the next section, this makes it easier to compare our results directly with theoretical predictions for this quantity.

Huang et al. (2013) also report multicomponent fits of a sample of 94 bright ETGs, but without imposing constraints based on simulations. From their analysis they identified three

\footnotetext{
4 This is by construction in the Cooper et al. models but obtained self-consistently in hydrodynamical simulations such as those of Rodriguez-Gomez et al. (2016).
}

subcomponents: a compact inner component, and intermediate component, and an outer and extended envelope. All the subcomponents in their work had average Sérsic indices $n \simeq 1-2$. In our work we imposed a restricted range of models using numerical simulations as "prior" or guide for the decomposition, simply to mitigate the degeneracy in multicomponent fits to ETG profiles. Nevertheless, our approach gives results that are fully consistent with Huang et al. (2013).

Moreover, looking at the rms scatter $\Delta$, of each fit, we can clearly see that by adding the third component we achieve an improvement of at least $10 \%$ for each galaxy. Since the expected value of $\Delta$ scales as $\sqrt{(m-k) / m}$ (see Seigar et al. 2007), where $m$ is the number of measured points ( $\sim 70$ in our case) and $k$ is the number of free parameters, we would need 18 free parameters to obtain an improvement of $10 \%$. This means that the improvement we obtain in our fits is not only due to the introduction of additional free parameters, as already shown by Seigar et al. (2007).

\section{Comparison with theoretical predictions for accreted mass fractions}

In the previous section, we identified inflections in the surface brightness profiles of galaxies in our sample that may correspond to transitions between regions dominated by debris from different accreted progenitors (or ensembles of progenitors) in different dynamical states. From our fitting procedure, we estimated the contributions of outer exponential "envelopes" to the total galaxy mass, which range from $28 \%$ to $60 \%$ for the galaxies in our sample, and the fraction of total accreted mass, which range from $83 \%$ to $95 \%$.

We can convert these results to estimates of the stellar mass fractions in the different components by assuming appropriate stellar mass-to-light ratios. To obtain typical colours for the central galaxies regions and for the outer envelopes, we measured the mean $g-i$ colour for each galaxy in regions where each of those components dominates. The mean colours are reported in Table 7 and are estimated for $R_{\mathrm{e}} \leq R \leq 2 R_{\mathrm{e}}$, where $R_{\mathrm{e}}$ is the effective radius derived by the two-component fit (see Table 4) for the inner and outer component.

We derived the mass-to-light ratios corresponding to the average colours, and hence the stellar mass of the whole galaxy, outer envelope, and accreted component, via the stellar population synthesis models (Vazdekis et al. 2012; Ricciardelli et al. 2012) with a Kroupa IMF. These results are summarized in Table 7. 
Table 7. Total and accreted stellar masses of galaxies in our sample.

\begin{tabular}{lccccccc}
\hline \hline Object & $\begin{array}{c}(g-i)_{\text {in }} \\
{[\mathrm{mag}]}\end{array}$ & $\begin{array}{c}(g-i)_{\text {out }} \\
{[\mathrm{mag}]}\end{array}$ & $\begin{array}{c}(M / L)_{\text {in }} \\
{\left[M_{\odot} / L_{\odot}\right]}\end{array}$ & $\begin{array}{c}(M / L)_{\text {out }} \\
{\left[M_{\odot} / L_{\odot}\right]}\end{array}$ & $\begin{array}{c}M_{\text {tot }}^{*} \\
{\left[M_{\odot}\right]}\end{array}$ & $\begin{array}{c}M_{\text {envelope }}^{*} \\
{\left[M_{\odot}\right]}\end{array}$ & $\begin{array}{c}M_{\text {total accreted }}^{*} \\
{\left[M_{\odot}\right]}\end{array}$ \\
\hline NGC 1399 & 1.2 & 0.8 & 5.2 & 0.6 & $5.8 \times 10^{11}$ & $3.7 \times 10^{11}$ & $4.9 \times 10^{11}$ \\
NGC 3923 & 1.3 & 1.4 & 6.2 & 9.1 & $1.5 \times 10^{12}$ & $4.9 \times 10^{11}$ & $1.2 \times 10^{12}$ \\
NGC 4365 & 1.1 & 0.9 & 3.4 & 0.9 & $4.3 \times 10^{11}$ & $1.5 \times 10^{11}$ & $3.4 \times 10^{11}$ \\
NGC 4472 & 1.2 & 1.1 & 5.2 & 3.4 & $8.6 \times 10^{11}$ & $2.3 \times 10^{11}$ & $7.8 \times 10^{11}$ \\
NGC 5044 & 1.2 & 0.7 & 5.2 & 0.5 & $5.7 \times 10^{11}$ & $2.3 \times 10^{11}$ & $4.5 \times 10^{11}$ \\
NGC 5846 & 1.4 & 0.7 & 9.1 & 0.5 & $9.6 \times 10^{11}$ & $5.8 \times 10^{11}$ & $8.5 \times 10^{11}$ \\
\hline
\end{tabular}

Notes. Columns 2-5 show the mean, extinction corrected, $(g-i)$ colour of the inner and outer regions of each galaxy and the relative mass-to-light ratios in the $g$ band. Columns 6 is the galaxy stellar mass, while Cols. 7 and 8 are the outer envelope and total accreted stellar masses, derived from the second component of the two-component fit and from both the accreted stellar masses in the three-component fit, respectively.

In Fig. 5 we compare the accreted mass ratios we infer from our observations (filled red triangles) with other observational estimates for BCGs by Seigar et al. (2007), Bender et al. (2015) and Iodice et al. (2016), theoretical predictions from semianalytic particle-tagging simulations by Cooper et al. (2013, 2015), and the Illustris cosmological hydrodynamical simulations (Rodriguez-Gomez et al. 2016). We find that the stellar mass fraction of the accreted component derived for galaxies in our sample is fully consistent both with published data for other BCGs (despite considerable differences in the techniques and assumptions involved) and with the theoretical models by Cooper et al. (2013, 2015).

In the same figure we also include the halo mass fraction obtained for NGC 1316, for which the VEGAS photometry will be published in a forthcoming paper (Iodice et al., in prep.). NGC 1316 is a peculiar galaxy in the Fornax cluster with a very perturbed morphology. The presence of shells, tidal tails, and dust indicates that the galaxy has undergone a recent interaction event. The accreted mass fraction and total stellar mass for this object are consistent with those of NGC 4472 and NGC 3923, which also show peculiar features, such as shells and dust lanes, and evidence of ongoing interactions.

In Fig. 5 we also compare the stellar mass fractions obtained for the outermost exponential component of our multicomponent fit (open red triangles) with the mass fraction associated with unbound debris streams from surviving cluster galaxies in the simulations of Cooper et al. (2015). We found that the mass fraction in this component of our fits is consistent with these values from the simulations, suggesting that such components may give a crude estimate of the mass distribution associated with dynamically unrelaxed components originating from disrupting or recently disrupted galaxies, as argued by Cooper et al. (2015).

\section{Discussion and conclusions}

We have presented new deep photometry in the $g$ and $i$ bands for four giant ETGs in the VST Early-type Galaxy Survey (VEGAS): NGC 3923, NGC 4365, NGC 5044, and NGC 5846. In particular, we studied the shapes of their surface brightness profiles to obtain evidence of structural variations that may constrain their assembly history. We also combined the results of this photometric study with those presented in Paper I, for NGC 4472, and in Iodice et al. (2016), for NGC 1399.

The data used were collected with the VST/OmegaCAM in March and April of 2015 within the Italian Guaranteed Time Observation (GTO). The large field of view (FOV) of OmegaCAM mounted on VST (one square degree), together with its high efficiency and spatial resolution, allows us to map the surface brightness of galaxies out to many effective radii and to very faint levels. We can therefore probe regions associated with the faint stellar envelopes of these galaxies, which, due to their long dynamical timescales, may retain signatures of their assembly history.

Our analysis suggests that the surface brightness profiles of the galaxies in our study are best reproduced by multicomponent models. As already pointed out by Mendez-Abreu et al. (2017), this kind of photometric decompositions need a "humansupervised" approach, and the choice of the number of components included in the fit is a relevant source of uncertainty. Since the surface brightness profiles of many galaxies could be fit equally well by a whole range of alternative models, the results of these photometric decompositions, especially for featureless early-type galaxies, strongly depends on the model adopted. We took two approaches to constructing such models. Adopting an empirically motivated, two-component approach most common in the literature, we find that four of our six galaxies (NGC 3923, NGC 4365, NGC 4472, and NGC 1399) can be modelled by an inner Sérsic component and an outer exponential, while the other two objects (NGC 5044 and NGC 5846) are best fitted with a double Sérsic model. The inner Sérsic components for each galaxy have an average effective radius $r_{\mathrm{e}} \sim 12 \mathrm{kpc}$ and Sérsic index $n \sim 4.3$, which is very similar to the values found (with a similar fitting philosophy) by Gonzalez et al. (2003) and Donzelli et al. (2011), i.e. $r_{\mathrm{e}} \sim 5-15 \mathrm{kpc}$ and $n \sim 4.4$.

We also used an alternative approach, which is motivated by the predictions of numerical simulations, in which we fitted the surface brightness profiles of our galaxies with three components: two dominant Sérsic components and an outer exponential component. To mitigate some of the degeneracy in this approach, we fixed the Sérsic index of the inner component to a representative value from simulations. In those simulations, this component corresponds to a mixture of stars formed in situ and the remnants of mergers are subject to violent relaxation. Compared to the traditional empirical fit, this approach allows us to make a more meaningful estimate of the total contribution of accreted stars. The accreted stellar mass fractions that we infer for galaxies in our sample with this approach are consistent with the theoretical predictions of Cooper et al. (2013), in which the fraction of accreted stars, and hence the Sérsic index of the overall surface brightness profile, increase strongly with stellar mass.

The most massive galaxies in our study, NGC 3923 and NGC 4472, are in the mass range typically identified with BCGs and ICL phenomena, as is NGC 1316 (Iodice et al., in prep.). These three galaxies show clear signs of ongoing interaction and 


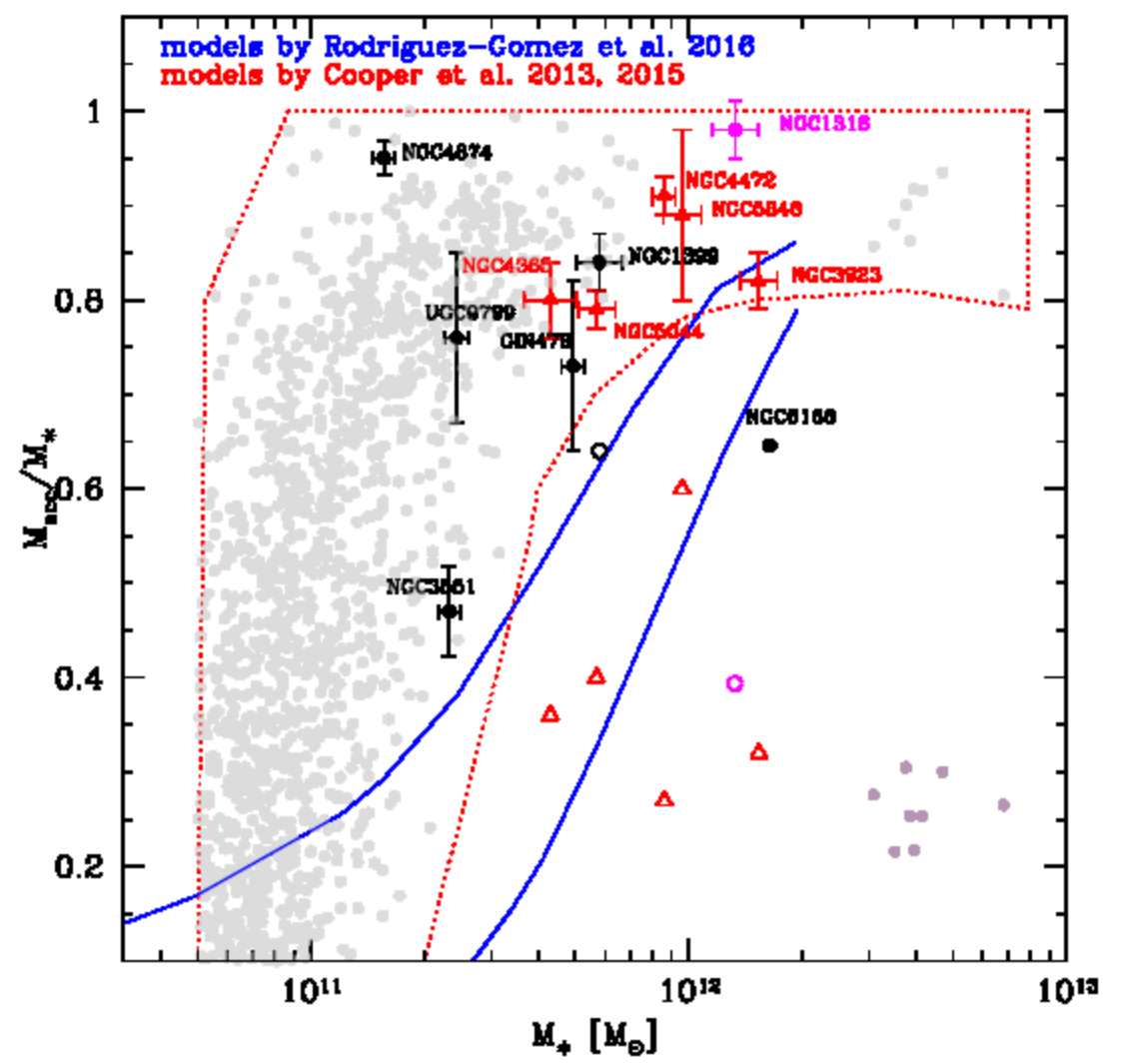

Fig. 5. Accreted mass fraction vs. total stellar mass for ETGs. Our VEGAS measurements are given as red filled and open triangles (see text for details). Black circles correspond to other BCGs from the literature (Seigar et al. 2007; Bender et al. 2015; Iodice et al. 2016) and the magenta dot represents NGC 1316 (Iodice et al., in prep.). Red and blue regions indicate the predictions of cosmological galaxy formation simulations by Cooper et al. $(2013,2015)$ and Rodriguez-Gomez et al. (2016), respectively. Purple-grey points show the mass fraction associated with the streams from Table 1 in Cooper et al. (2015) for comparison to the observations shown by open symbols.

recent accretion events, indicating that their outer regions are still being assembled, which is consistent with theoretical expectations for such galaxies (e.g. Cooper et al. 2015). In this regard it is interesting that the mass fractions in the exponential components of our two-component profile decompositions are in good agreement with the mass fractions associated with streams from surviving galaxies in the simulations of Cooper et al. (2015). This suggests these outer exponential components may give a crude estimate of the stellar mass fraction associated with recently disrupted galaxies. We find values for this fraction ranging from $28 \%$ to $64 \%$.

Previous works, for example by Gonzalez et al. (2005) and Zibetti et al. (2005), have identified outer components similar to those in our fits with so-called diffuse intracluster light (ICL). Typical claims for the mass fractions in ICL range from 10-30\%. From a theoretical perspective, it is not clear that it is physically meaningful to attempt to distinguish such a component from the outer envelopes of BCGs (see for example the discussion in Cooper et al. 2015). A wide variety of definitions of ICL have been adopted in both the observational and theoretical literature, and according to many of these studies, such components cannot be unambiguously identified from photometry alone. We therefore leave open the question of how the outer-component light fractions we derive relate in detail to previous claims regarding the nature of ICL.

For all the galaxies in our study we can identify at least one inflection in the surface brightness profile. These inflections occur at very faint surface brightness levels $\left(24.0 \leq \mu_{g} \leq\right.$ $\left.27.8 \mathrm{mag} / \operatorname{arcsec}^{2}\right)$. They appear to correlate with changes in the trend of ellipticity, position angle, and colour with radius, where the isophotes become flatter and misaligned and the colours become bluer beyond the inflections. This suggests that these inflections mark transitions between physically distinct components (or ensembles of similar components) in different states of dynamical relaxation. This sense of the surface brightness inflection is upward (i.e. a break to a shallower slope) in NGC 3923, NGC 4365, and NGC 4472, and downward (i.e. a break to a steeper slope) in the other galaxies.

A variety of possible interpretations for such features have been suggested based on theoretical models. Upward inflections (shallower outer slopes) might reflect transitions between either an inner in situ-dominated region and an outer accretiondominated region, or else between two accreted components (Abadi et al. 2006; Font et al. 2011). Downward inflections can occur within the profiles of debris from a single progenitors alone, corresponding to the characteristic apocentric radius of its stars (Cooper et al. 2010; Deason et al. 2013; Amorisco 2017) and therefore need not represent a transition between two separate components; however they need not always correspond to a single progenitor because they can also be created by a drop in the density of only one of a small number of contributions having a roughly equal density interior to the break. It has been claimed that the strength and radius of downward inflections in the accreted component encode information on the number of progenitors as well as the orbital properties of the dominant progenitor and its disruption timescale. For example, Deason et al. (2013) examine aspects of these relationships in the context of satellite accretion onto Milky Way analogues and claim that the 
properties of the break can be used to infer the accretion time of the progenitor. In massive ETGs the picture is further complicated by violent relaxation of the central potential (e.g. Hilz et al. 2012).

Since there are many ways to produce these inflections, any given profile might in principle be expected to have several inflections in both directions. In practice (as seen in the profile decompositions in Cooper et al. 2010, 2015) the fact that the accreted component is the superposition of many contributions tends to blur out most of the individual dynamical downward inflections, but nevertheless to preserve a (more gradual) average steepening of the profile to larger radius. In support of such predictions, D'Souza et al. (2014) found that the transition radius between the different accreted components, $R_{\mathrm{tr}}$, varies systematically with the stellar mass, which is on average smaller for more massive galaxies, in agreement with the trends predicted by Cooper et al. (2013), Rodriguez-Gomez et al. (2016). We also find a possible correlation between the slope of the surface brightness profiles beyond the inflection point and the density of the field around each galaxy, indicating that objects in more dense fields (hence perhaps more massive dark matter halos) have steeper outer profiles.

For our galaxies with upward profile inflections, the inflection point is well beyond $2 R_{\mathrm{e}}$, suggesting that these features are attributable to an excess in the number of weakly bound stars with large orbital apocentres, perhaps associated with more recent accretion events. We do not observe significant changes in surface brightness or other properties at the transition point between the inner two components of our theoretically motivated three-component fits. This is perhaps not surprising because simulations predict that the in situ accreted transition is hardly noticeable in very massive galaxies, for the most part because the in situ stars account for a relatively small fraction of the total mass even in the bright body of the galaxy. In the most massive galaxies, the accreted mass is expected to be made up from $\sim 10$ significant contributions with roughly similar mass, and of a similar mass to the in situ component; this is in contrast to Milky Way-mass galaxies, which are expected to have stellar halos dominated by only one or two progenitors much less massive than the in situ contribution (Cooper et al. 2013). Moreover the same models predict that the ages and metallicities of in situ stars in massive ETGs are quite similar to those of the most significant "relaxed" accreted components (De Lucia \& Blaizot 2007; Cooper et al. 2013).

It is encouraging that we see a variety of profile inflections in our photometric investigation of this small subset of the VEGAS sample and that these are broadly consistent with the expectations of state-of-the-art theoretical models. Our results suggests that with the complete sample of extremely deep surface brightness profiles from the full survey, we will be able to investigate the late stages of massive galaxy assembly statistically, thereby distinguishing dynamically evolved systems from those that are still reaching dynamical equilibrium and probing the balance between in situ star formation and accretion across a wide range of stellar mass. This is a promising route to constraining cosmological models of galaxy formation such as those we have compared with here, which predict fundamental, relatively tight correlations between the present-day structure of massive galaxies and the growth histories of their host dark matter halos.

Acknowledgements. This work is based on visitor mode observations taken at the ESO La Silla Paranal Observatory within the VST Guaranteed Time Observations, Programme IDs 090.B-0414(D), 091.B-0614(A), 094.B- 0496(A), 094.B-0496(B), 094.B-0496(D) and 095.B-0779(A). The authors wish to thank the anonymous referee for his or her comments and suggestions that allowed us to greatly improve the paper. The authors wish to thank ESO for the financial contribution given for the visitor mode runs at the ESO La Silla Paranal Observatory. M. Spavone wishes to thank the ESO staff of the Paranal Observatory for their support during the observations at VST. A.P.C. is supported by a COFUND/Durham Junior Research Fellowship under EU grant [267209] and acknowledges support from STFC (ST/L00075X/1). The data reduction for this work was carried out with the computational infrastructure of the INAF-VST Center at Naples (VSTceN). This research made use of the NASA/IPAC Extragalactic Database (NED), which is operated by the Jet Propulsion Laboratory, California Institute of Technology, under contract with the National Aeronautics and Space Administration, and has been partly supported by the PRININAF "Galaxy evolution with the VLT Survey Telescope (VST)" (P.I. A. Grado). N.R.N., E.I., and M.P. have been supported by the PRIN-INAF 2014 "Fornax Cluster Imaging and Spectroscopic Deep Survey" (PI. N.R. Napolitano). M.S., E.I., and M. Cantiello acknowledge finacial support from the VST project (P.I. M. Capaccioli).

\section{References}

Abadi, M. G., Navarro, J. F., \& Steinmetz, M. 2006, MNRAS, 365, 747 Amorisco, N. C. 2017, MNRAS, 464, 2882

Ann, H. B. 2010, in Galaxies and their Masks, eds. D. L., Block, K. C. Freeman, \& I. Puerari (Springer Science + Business Media), 195

Arnaboldi, M., Ventimiglia, G., Iodice, E., Gerhard, O., \& Coccato, L. 2012, A\&A, 545, A37

Arnold, J. A., Romanowsky, A. J., Brodie, J. P., et al. 2014, ApJ, 791, 80 Arrigoni Battaia, F., Gavazzi, G., Fumagalli, M., et al. 2012, A\&A, 543, A112 Bender, R., Surma, P., Doebereiner, S., Moellenhoff, C., \& Madejsky, R. 1989, A\&A, 217, 35

Bender, R., Kormendy, J., Cornell, M. E., \& Fisher, D. B. 2015, ApJ, 807, 56

Bertin, E., \& Arnouts, S. 1996, A\&AS, 117, 393

Binney, J., \& de Vaucouleurs, G. 1981, MNRAS, 194, 679

Binney, J., \& Merrifield, M. 1998, Galactic astronomy/James Binney and Michael Merrifield (Princeton, NJ: Princeton University Press)

Blakeslee, J. P., Jordán, A., Mei, S., et al. 2009, ApJ, 694, 556

Blom, C., Spitler, L. R., \& Forbes, D. A. 2012a, MNRAS, 420, 37

Blom, C., Forbes, D. A., Brodie, J. P., et al. 2012b, MNRAS, 426, 1959

Blom, C., Forbes, D. A., Foster, C., Romanowsky, A. J., \& Brodie, J. P. 2014, MNRAS, 439, 2420

Bogdán, Á., Forman, W. R., Zhuravleva, I., et al. 2012, ApJ, 753, 140

Buote, D. A., \& Canizares, C. R. 1998, MNRAS, 298, 811

Buote, D. A., Lewis, A. D., Brighenti, F., \& Mathews, W. G. 2003, ApJ, 594, 741

Burstein, D., \& Heiles, C. 1982, AJ, 87, 1165

Caon, N., Capaccioli, M., \& D'Onofrio, M. 1993, MNRAS, 265, 1013

Caon, N., Capaccioli, M., \& D’Onofrio, M. 1994, A\&AS, 106, 199

Capaccioli, M., \& de Vaucouleurs, G. 1983, ApJS, 52, 465

Capaccioli, M., \& Schipani, P. 2011, The Messenger, 146, 2

Capaccioli, M., Caon, N., \& D'Onofrio, M. 1992, MNRAS, 259, 323

Capaccioli, M., Spavone, M., Grado, A., et al. 2015, A\&A, 581, A10

Carter, D., Thomson, R. C., \& Hau, G. K. T. 1998, MNRAS, 294, 182

Ciotti, L., \& Bertin, G. 1999, A\&A, 352, 447

Colbert, J. W., Mulchaey, J. S., \& Zabludoff, A. I. 2001, AJ, 121, 808

Cole, S., Lacey, C. G., Baugh, C. M., \& Frenk, C. S. 2000, MNRAS, 319, 168

Cooper, A. P., Cole, S., Frenk, C. S., et al. 2010, MNRAS, 406, 744

Cooper, A. P., D’Souza, R., Kauffmann, G., et al. 2013, MNRAS, 434, 3348

Cooper, A. P., Gao, L., Guo, Q., et al. 2015, MNRAS, 451, 2703

Davies, R. L., Kuntschner, H., Emsellem, E., et al. 2001, ApJ, 548, L33

Deason, A. J., Belokurov, V., Evans, N. W., \& Johnston, K. V. 2013, ApJ, 763, 113

De Lucia, G., \& Blaizot, J. 2007, MNRAS, 375, 2

De Lucia, G., Springel, V., White, S. D. M., Croton, D., \& Kauffmann, G. 2006,

MNRAS, 366, 499

D’Onofrio, M., Capaccioli, M., \& Caon, N. 1994, MNRAS, 271, 523

Donzelli, C. J., Muriel, H., \& Madrid, J. P. 2011, ApJS, 195, 15

D’Souza, R., Kauffman, G., Wang, J., \& Vegetti, S. 2014, MNRAS, 443, 1433

Duc, P.-A., Cuillandre, J.-C., Karabal, E., et al. 2015, MNRAS, 446, 120

de Vaucouleurs, G., \& Capaccioli, M. 1979, ApJS, 40, 699

Faltenbacher, A., \& Mathews, W. G. 2005, MNRAS, 362, 498

Ferrarese, L., Côté, P., Jordán, A., et al. 2006, ApJS, 164, 334

Ferrarese, L., Côté, P., Cuillandre, J.-C., et al. 2012, ApJS, 200, 4

Font, A. S., McCarthy, I. G., Crain, R. A., et al. 2011, MNRAS, 416, 2802

Foster, C., Pastorello, N., Roediger, J., et al. 2016, MNRAS, 457, 147

Frenk, C. S., White, S. D. M., Efstathiou, G., \& Davis, M. 1985, Nature, 317, 595

Gastaldello, F., Buote, D. A., Temi, P., et al. 2009, ApJ, 693, 43 
Gonzalez, A. H., Zabludoff, A. I., \& Zaritsky, D. 2003, Ap\&SS, 285, 67 Gonzalez, A. H., Zabludoff, A. I., \& Zaritsky, D. 2005, ApJ, 618, 195

Grado, A., Capaccioli, M., Limatola, L., \& Getman, F. 2012, Mem. Soc. Astron. Ital. Suppl., 19, 362

Guo, Q., \& White, S. D. M. 2008, MNRAS, 384, 2

Hamabe, M., \& Kormendy, J. 1987, in Structure and Dynamics of Elliptical Galaxies, IAU Symp., 127, 379

Haynes, M. P., \& Giovanelli, R. 1991, AJ, 102, 841

Hilz, M., Naab, T., Ostriker, J. P., et al. 2012, MNRAS, 425, 3119

Huang, Z., Radovich, M., Grado, A., et al. 2011, A\&A, 529, A93

Huang, S., Ho, L. C., Peng, C. Y., Li, Z.-Y., \& Barth, A. J. 2013, ApJ, 766, 47

Iodice, E., Capaccioli, M., Grado, A., et al. 2016, ApJ, 820, 42

Irwin, J. A., \& Sarazin, C. L. 1996, ApJ, 471, 683

Janowiecki, S., Mihos, J. C., Harding, P., et al. 2010, ApJ, 715, 972

Jedrzejewski, R. I. 1987, MNRAS, 226, 747

Johnston, K. V., Bullock, J. S., Sharma, S., et al. 2008, ApJ, 689, 936

Kauffmann, G., White, S. D. M., \& Guiderdoni, B. 1993, MNRAS, 264, 201

Kim, E., Lee, M. G., \& Geisler, D. 2000, MNRAS, 314, 307

Kormendy, J. 1977, ApJ, 218, 333

Kormendy, J., Fisher, D. B., Cornell, M. E., \& Bender, R. 2009, ApJS, 182, 216

Kronawitter, A., Saglia, R. P., Gerhard, O., \& Bender, R. 2000, A\&AS, 144, 53

La Barbera, F., De Carvalho, R. R., De La Rosa, I. G., et al. 2010, AJ, 140, 1528

Longobardi, A., Arnaboldi, M., Gerhard, O., \& Hanuschik, R. 2015a, A\&A, 579, A135

Longobardi, A., Arnaboldi, M., Gerhard, O., \& Mihos, J. C. 2015b, A\&A, 579, L3

Lucy, L. B. 1974, AJ, 79, 745

MacArthur, L. A., Courteau, S., \& Holtzman, J. A. 2003, ApJ, 582, 689

Malin, D. F., \& Carter, D. 1980, Nature, 285, 643

Mei, S., Blakeslee, J. P., Côté, P., et al. 2007, ApJ, 655, 144

Mendel, J. T., Proctor, R. N., Forbes, D. A., \& Brough, S. 2008, MNRAS, 389, 749
Mendez-Abreu, J., Ruiz-Lara, T., Sanchez-Menguiano, L., et al. 2017, A\&A, 598, A32

Mihos, J. C., Harding, P., Rudick, C. S., \& Feldmeier, J. J. 2013, ApJ, 764, L20 Oser, L., Ostriker, J. P., Naab, T., Johansson, P. H., \& Burkert, A. 2010, ApJ, 725, 2312

Paolillo, M., Fabbiano, G., Peres, G., \& Kim, D.-W. 2002, ApJ, 565, 883 Peng, E. W., Jordán, A., Côté, P., et al. 2006, ApJ, 639, 95

Pillepich, A., Vogelsberger, M., Deason, A., et al. 2014, MNRAS, 444, 237

Pohlen, M., \& Trujillo, I. 2006, A\&A, 454, 759

Purcell, C. W., Bullock, J. S., \& Zentner, A. R. 2007, ApJ, 666, 20

Ricciardelli, E., Vazdekis, A., Cenarro, A. J., \& Falcón-Barroso, J. 2012, MNRAS, 424, 172

Richardson, W. H. 1972, J. Opt. Soc. Am. (1917-1983), 62, 55

Rodriguez-Gomez, V., Pillepich, A., Sales, L. V., et al. 2016, MNRAS, 458, 2371

Sandage, A., \& Bedke, J. 1994, The Carnegie Atlas of Galaxies, Vol. I, II, eds. A. Sandage, \& J. Bedke (Carnegie Institution of Washington Publ.), 638

Seigar, M. S., Graham, A. W., \& Jerjen, H. 2007, MNRAS, 378, 1575

Schlegel, D. J., Finkbeiner, D. P., \& Davis, M. 1998, ApJ, 500, 525

Sérsic, J. L. 1963, Boletin de la Asociacion Argentina de Astronomia La Plata Argentina, 6, 41

Sikkema, G., Carter, D., Peletier, R. F., et al. 2007, A\&A, 467, 1011

Surma, P. 1993, in Structure, Dynamics and Chemical Evolution of Elliptical Galaxies, ESO, Conference and Workshop Proc., 45, 669

Tonry, J. L., Dressler, A., Blakeslee, J. P., et al. 2001, ApJ, 546, 681

Tortora, C., Napolitano, N. R., Cardone, V. F., et al. 2010, MNRAS, 407, 144

Trinchieri, G., \& Goudfrooij, P. 2002, A\&A, 386, 472

Vazdekis, A., Ricciardelli, E., Cenarro, A. J., et al. 2012, MNRAS, 424, 157

Villumsen, J. V. 1983, MNRAS, 204, 219

White, S. D. M., \& Frenk, C. S. 1991, ApJ, 379, 52

Zibetti, S., White, S. D. M., Schneider, D. P., \& Brinkmann, J. 2005, MNRAS, 358, 949 


\section{Appendix A: Masking of bright sources}
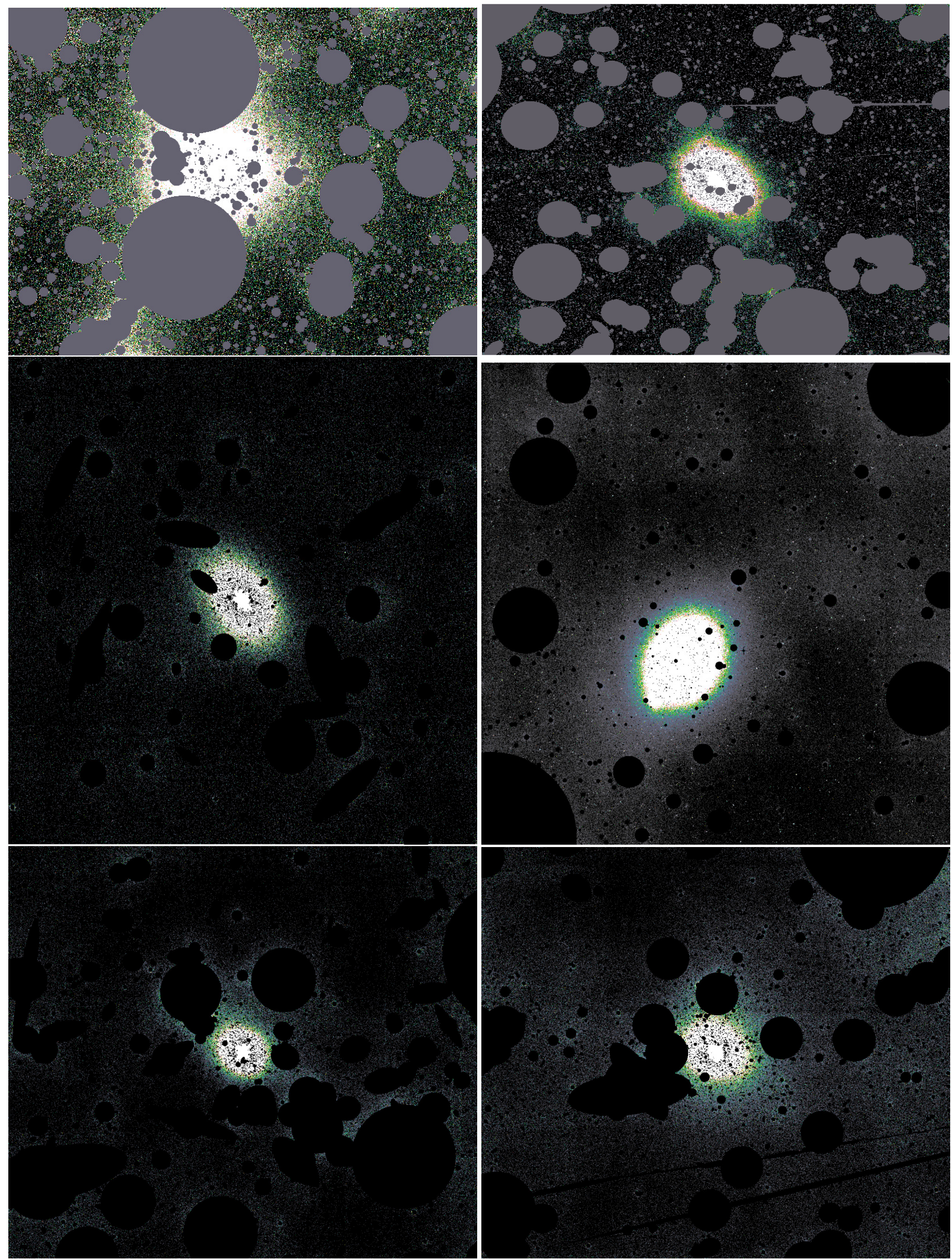

Fig. A.1. VST $g$-band mosaic of NGC 1399, NGC 3923, NGC 4365, NGC 4472, NGC 5044, and NGC 5846, showing the masking of bright sources in the field. 
In Fig. A.1, we show the masks used in this work for the photometric analysis of NGC 1399, NGC 3923, NGC 4365, NGC 4472, NGC 5044, and NGC 5846, respectively. All sources have been masked out by using $\operatorname{ExAM}^{5}$ (Huang et al. 2011), a programme based on SExtractor (Bertin \& Arnouts 1996), which was developed to accurately mask background and foreground sources, reflection haloes, and spikes from saturated stars.

\section{Appendix B: Surface brightness profiles in azimuthal sectors}

We measured the surface brightness profiles for each galaxy in four azimuthal sectors to test the reliability of the profile measurements at large galaxy radii and to quantify the combined systematic uncertainties due to masking, assumption of elliptical isophotes, stray light, etc. The same has already been already done for NGC 1399 by Iodice et al. (2016).
We found that the surface brightness profiles in the outskirts of almost all galaxies are on average the same as the azimuthally averaged profiles. The only exception is NGC 5044 in the SW sector, where it shows an excess of light. Such excess is observed at bright magnitudes $\left(\sim 25 \mathrm{mag} / \mathrm{arcsec}^{2}\right)$ and it is in the direction where most of the galaxies belonging to the group are located. Since NGC 5044 belongs to a fossil group of galaxies (Faltenbacher \& Mathews 2005), the excess of light observed in the SW direction could be the signature of the presence of intracluster light.

\footnotetext{
5 ExAM is a code developed by $\mathrm{Z}$. Huang during his $\mathrm{PhD}$. A detailed description of the code can be found in his Ph.D. thesis, available at the following link: http://www. fedoa.unina.it/id/eprint/8368
} 

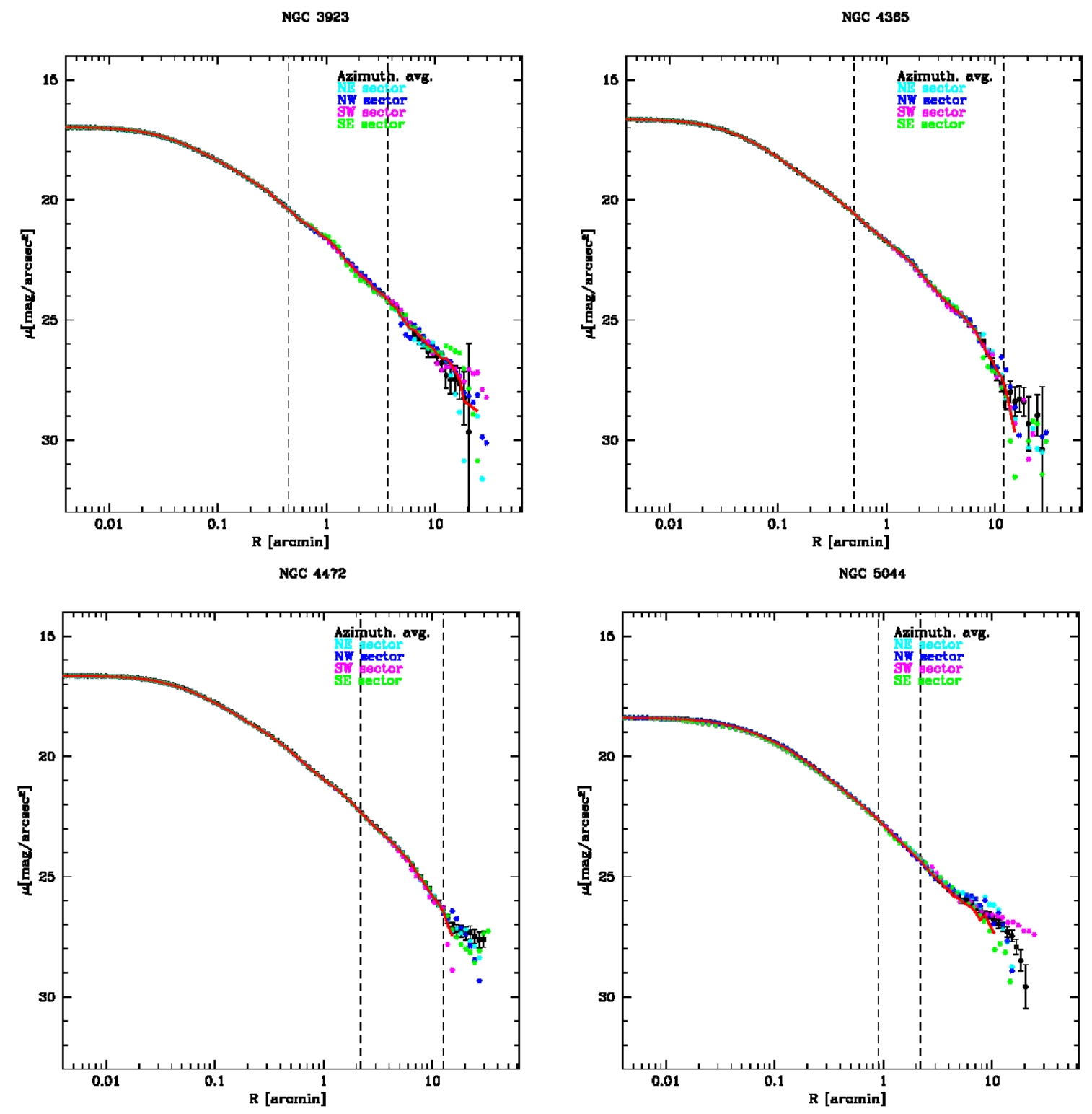

NGC 5848

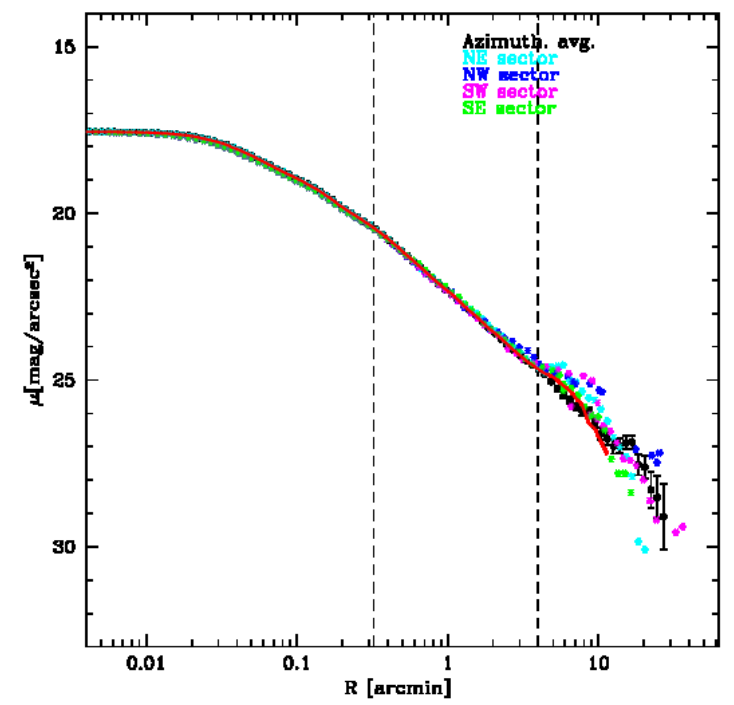

Fig. B.1. VST $g$-band surface brightness profiles of NGC 3923, NGC 4365, NGC 4472, NGC 5044, and NGC 5846, extracted in azimuthal sectors and compared with the azimuthally averaged profile. The red line is the average profile, while the dashed lines indicate the location of the transition radii. 


\section{Appendix C: Two-component models fixing the innnermost (in situ) component}

In Sect. 5.3 we presented three-component fits to our sample of ETG light profiles using a theoretically motivated constraint on the Sersic index of the innermost component (representing stars formed in situ) and the outermost component (representing unrelaxed debris). In Fig. C.1 and Table C.1 we present analogous results for the dominant component (representing relaxed accreted stars) when we do not include an exponential outer component. The parameters obtained in this way are more directly comparable to those of other work using two-component fitting (e.g., in particular, the higher values of $n_{2}$ ), but they are less representative of the behaviour of the outermost part of the profiles in our deep observations.

Table C.1. Best-fitting structural parameters for a two-component fit in which $n$ for the inner (in situ) component is fixed.

\begin{tabular}{|c|c|c|c|c|c|c|c|c|c|}
\hline Object & $\begin{array}{c}\mu_{\mathrm{e} 1} \\
{\left[\mathrm{mag} / \operatorname{arcsec}^{2}\right]}\end{array}$ & $\begin{array}{c}r_{\mathrm{e} 1} \\
{[\operatorname{arcsec}]}\end{array}$ & $n_{1}$ & $\begin{array}{c}\mu_{\mathrm{e} 2} \\
{\left[\mathrm{mag} / \operatorname{arcsec}^{2}\right]}\end{array}$ & $\begin{array}{c}r_{\mathrm{e} 2} \\
{[\operatorname{arcsec}]}\end{array}$ & $n_{2}$ & $\begin{array}{c}m_{\mathrm{T}, 1} \\
{[\mathrm{mag}]}\end{array}$ & $\begin{array}{c}m_{\mathrm{T}, 2} \\
{[\mathrm{mag}]}\end{array}$ & $f_{h}$ \\
\hline & 760 & & 2 & 20 & & & & 8.30 & \\
\hline & & & 2 & & & & & & $84 \%$ \\
\hline & & & 2 & & & & & & $90 \%$ \\
\hline & & 50 & 2 & & $547 \pm$ & & & & $79 \%$ \\
\hline NGC 5044 & $22.16 \pm 0.05$ & $39.3 \pm 1.8$ & 2.5 & $27.03 \pm 0.17$ & $678 \pm 55$ & $1.54 \pm 0.27$ & 10.80 & 9.49 & $77 \%$ \\
\hline NGC 5846 & $21.11 \pm 0.16$ & $18.9 \pm 1.1$ & 2 & $26.87 \pm 0.28$ & $757 \pm 101$ & $5.47 \pm 0.68$ & 11.34 & 9.09 & $89 \%$ \\
\hline
\end{tabular}

Notes. Columns 2-4 report effective magnitude, effective radius, and Sérsic index for the inner component of each fit. The Sérsic index for the in situ component has been fixed to $n \sim 2$ using the models as a prior (Cooper et al. 2013). We allowed small variations of \pm 0.5 around the mean value of $n=2$. This would bracket the range of $n$ in the simulations and allows us to obtain a better fit. Columns 5-7 list the same parameters for the outer Sérsic component. Columns 8 and 9 report the total magnitude of the inner $\left(m_{\mathrm{T}, 1}\right)$ and outer Sérsic component $\left(m_{\mathrm{T}, 2}\right)$. Column 12 gives the relative fraction of the outer component with respect to the total luminosity of the galaxy. 
NGC 1399
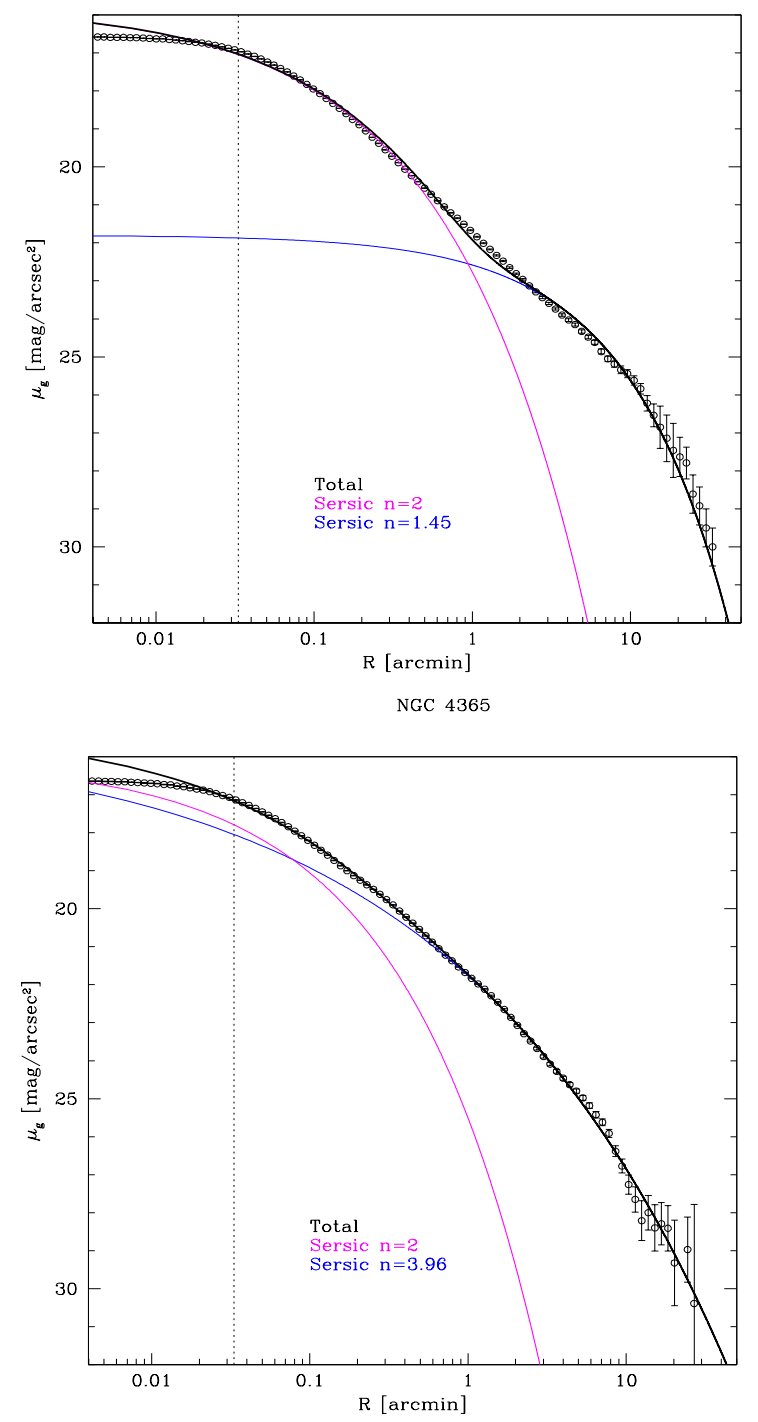

NGC 5044

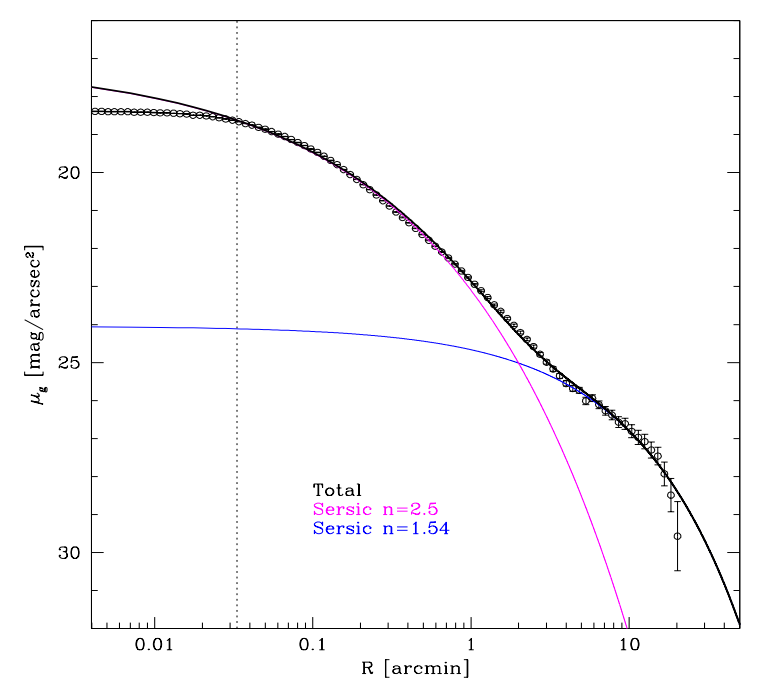

NGC 3923
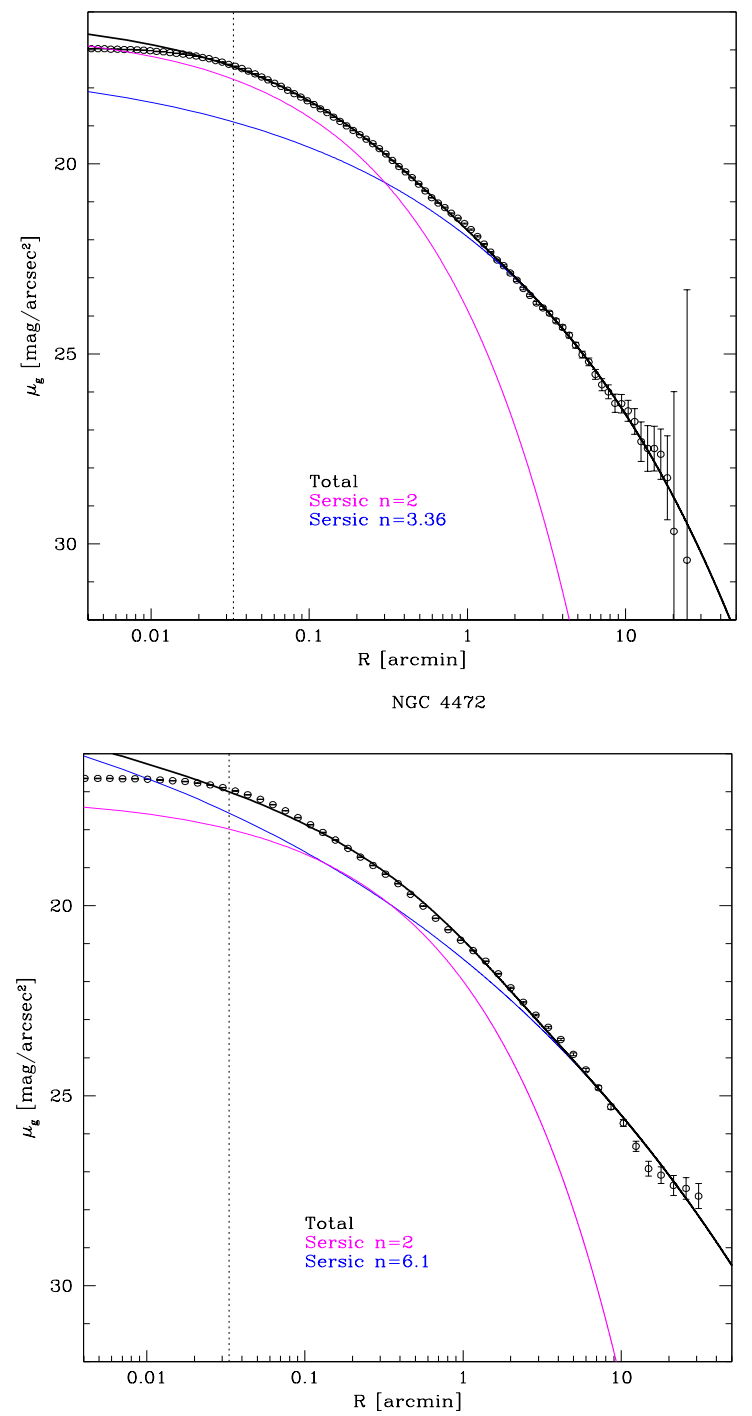

NGC 5846

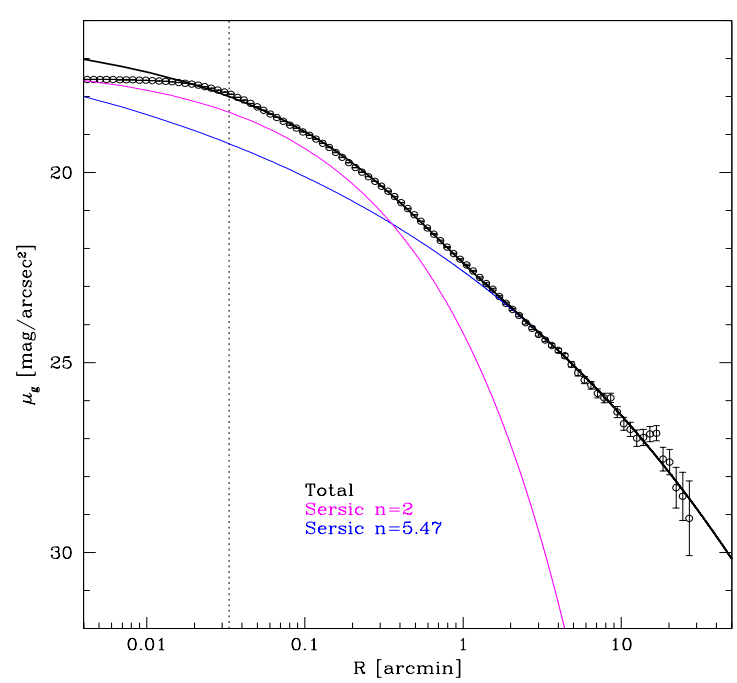

Fig. C.1. VST $g$-band profiles of NGC 1399, NGC 3923, NGC 4365, NGC 4472, NGC 5044, and NGC 5846 plotted on a logarithmic scale. The blue line indicates a fit to the outer regions. The magenta line indicates a fit to the inner regions with a Sérsic profile with $n$ fixed from theory (Cooper et al. 2013), and the black line indicates the sum of the components in each fit. The dotted line indicates the core of the galaxy $(R \sim 2$ arcsec), which was excluded in the fit. 


\section{Appendix D: Additional figures}
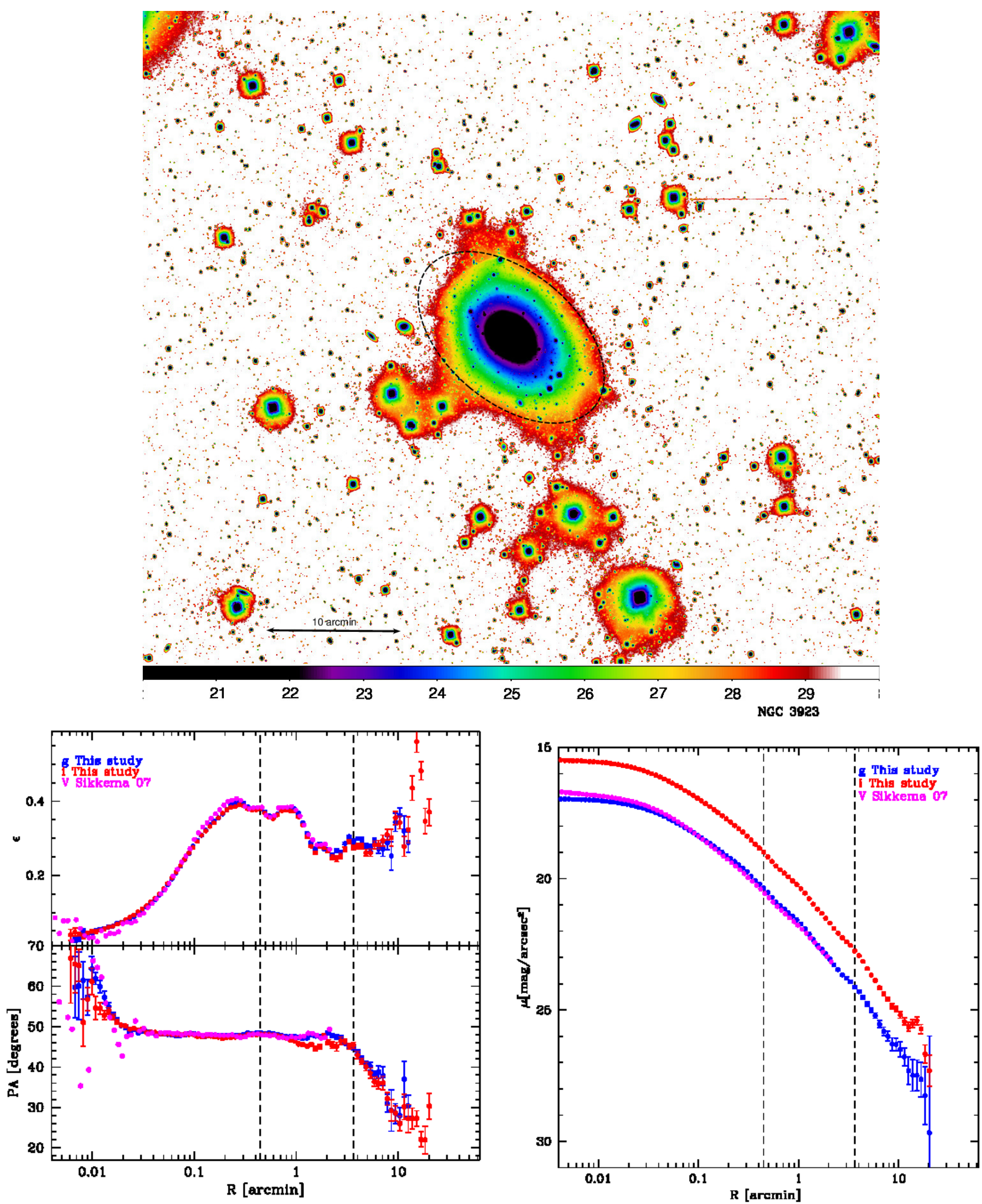

Fig. D.1. Same as Fig. 1 for NGC 3923. The $V$-band measurements of Sikkema et al. (2007; magenta) shifted by +0.7 mag to match the amplitude of the VEGAS profile. 

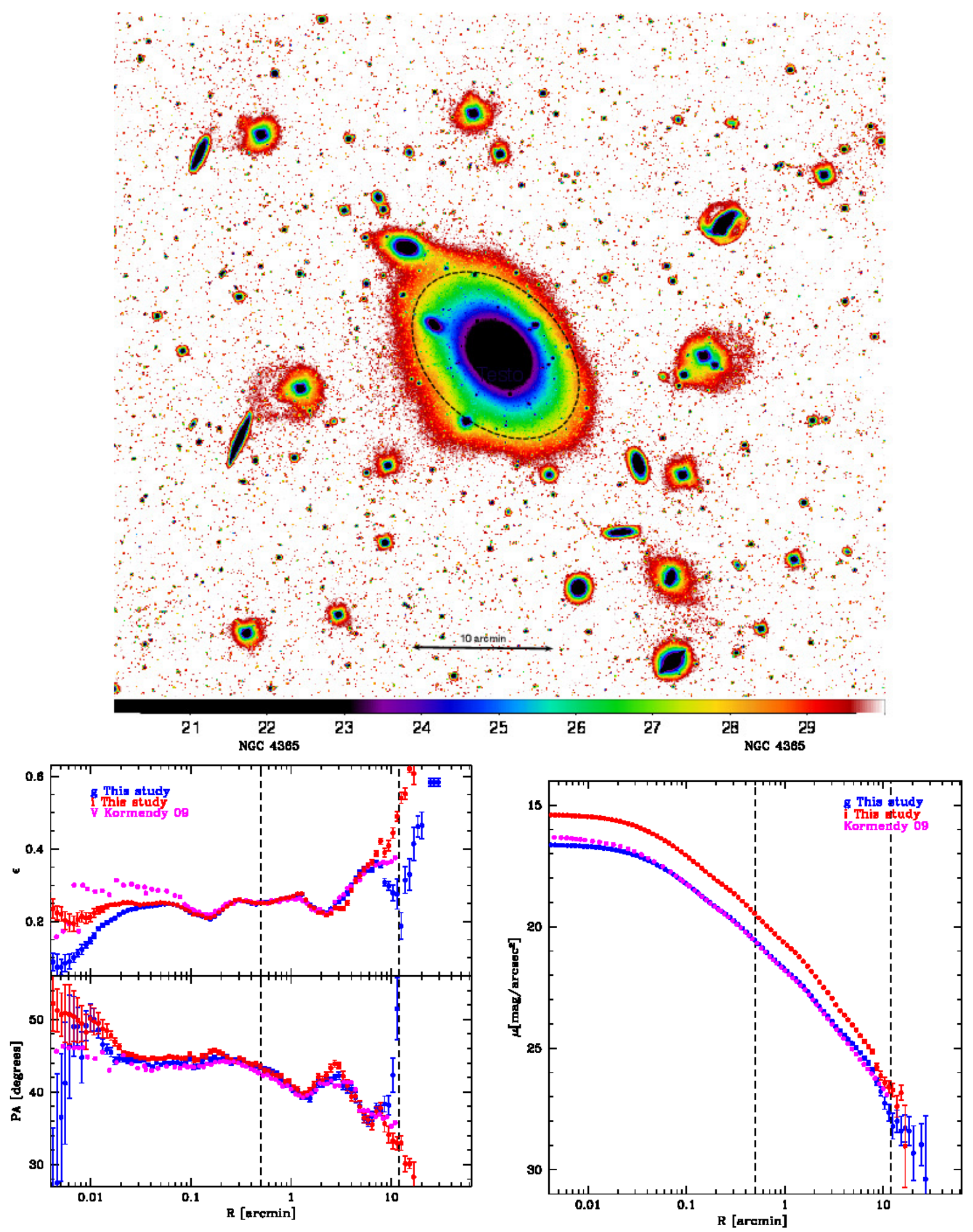

Fig. D.2. Same as Fig. D.1 for NGC 4365. The $V$-band surface brightness profile by Kormendy et al. (2009, magenta points in the bottom panel) was shifted by +0.2 mag to match the amplitude of the VEGAS profile. 
M. Spavone et al.: Stellar halos of giant ETGs
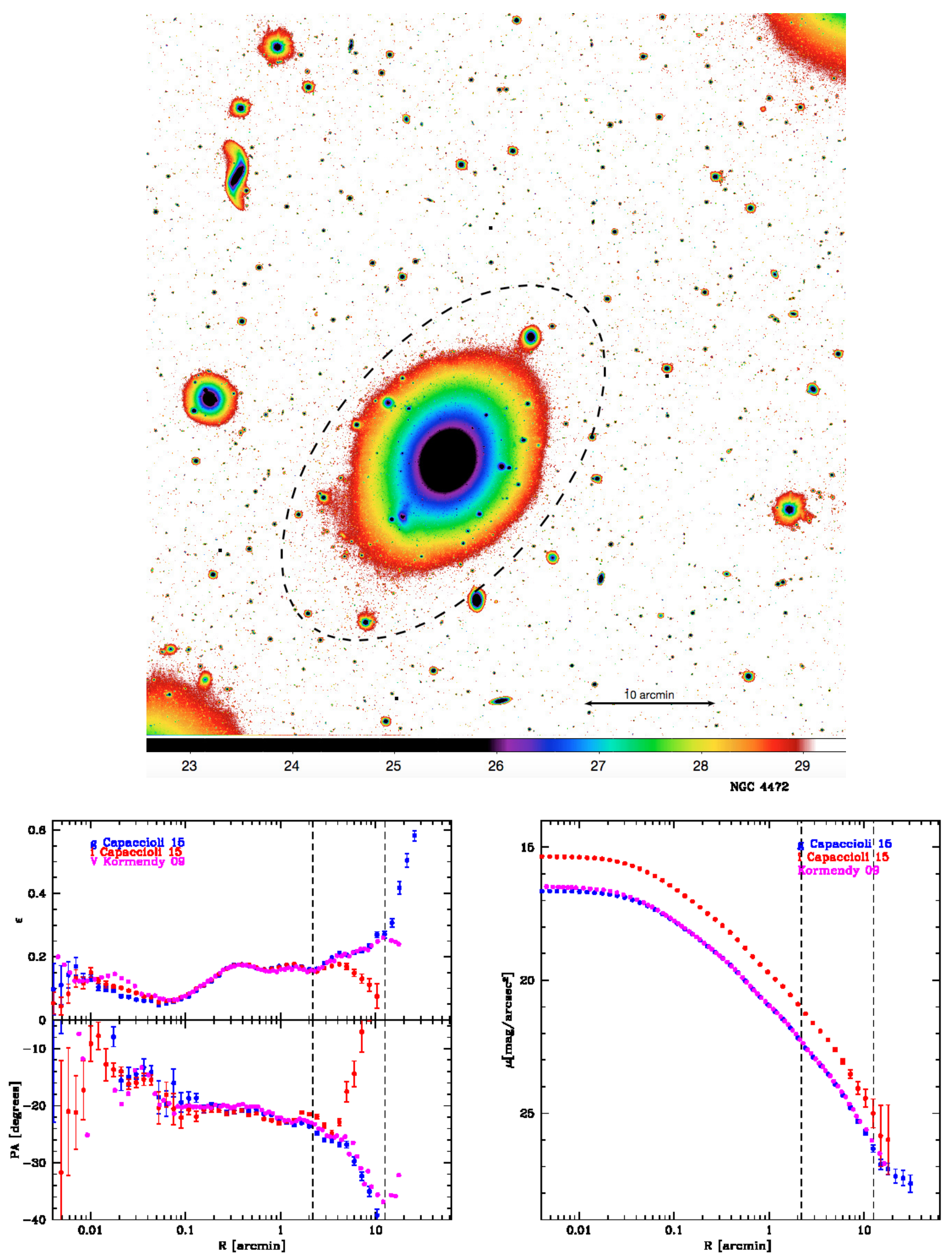

Fig. D.3. Same as Fig. D.1 for NGC 4472. The $V$-band surface brightness profile by Kormendy et al. (2009, magenta points in the bottom panel) was shifted by +0.33 mag to match the amplitude of the VEGAS profile. 

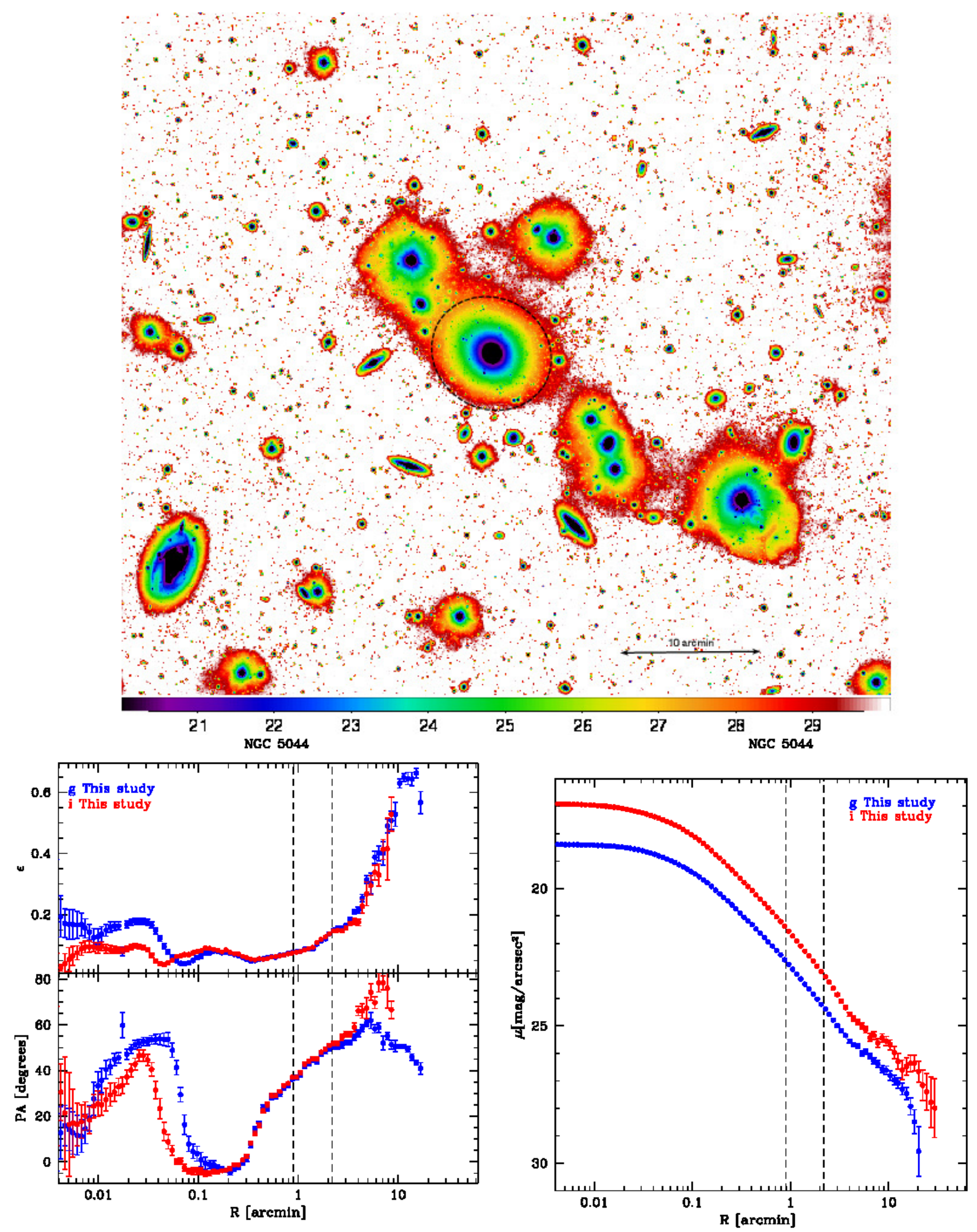

Fig. D.4. Same as Fig. D.1 for NGC 5044. 
M. Spavone et al.: Stellar halos of giant ETGs
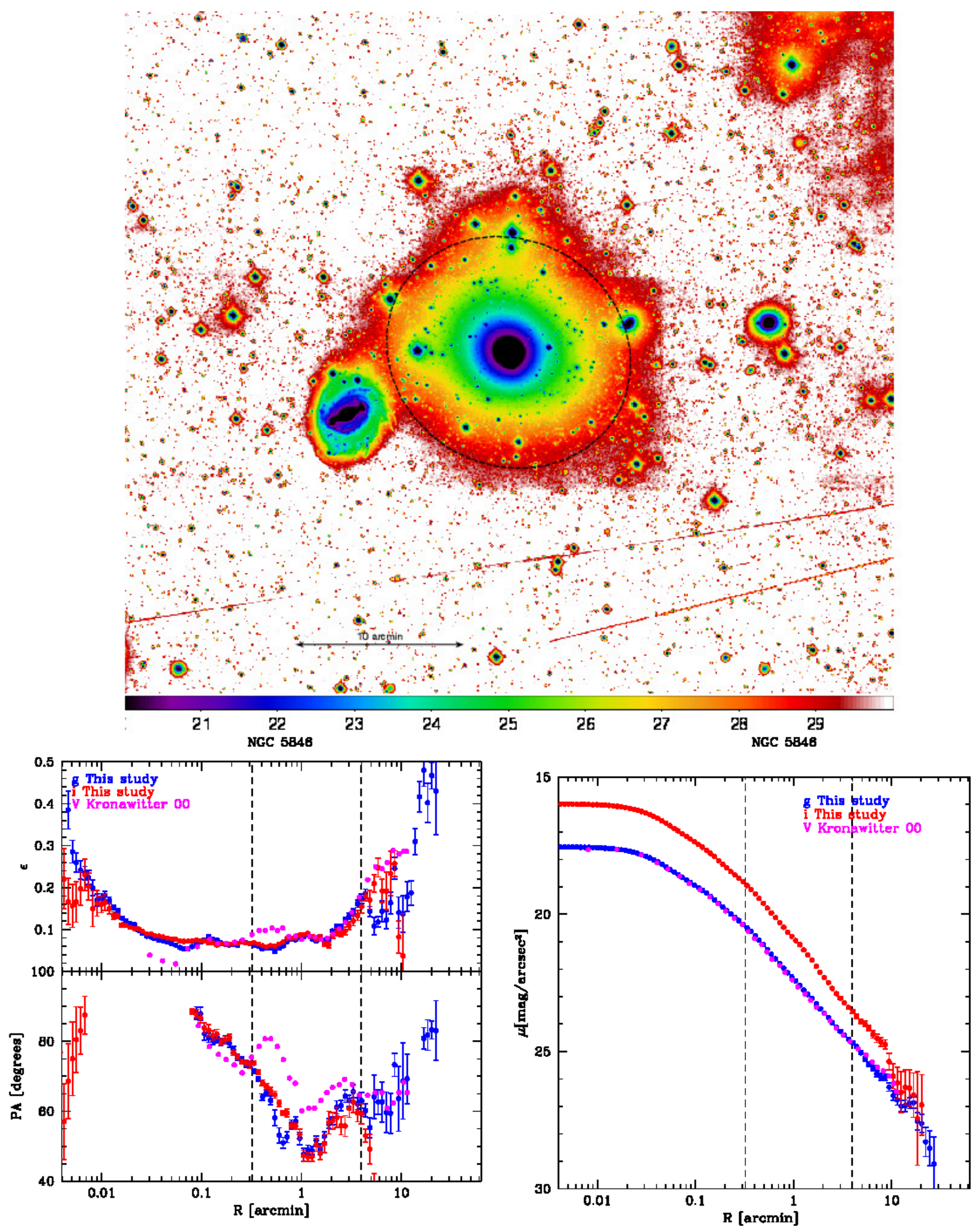

Fig. D.5. Same as Fig. D.1 for NGC 5846. The $V$-band surface brightness profile by Kronawitter et al. (2000, magenta points in the bottom panel) was shifted by +0.23 mag to match the VEGAS result. 

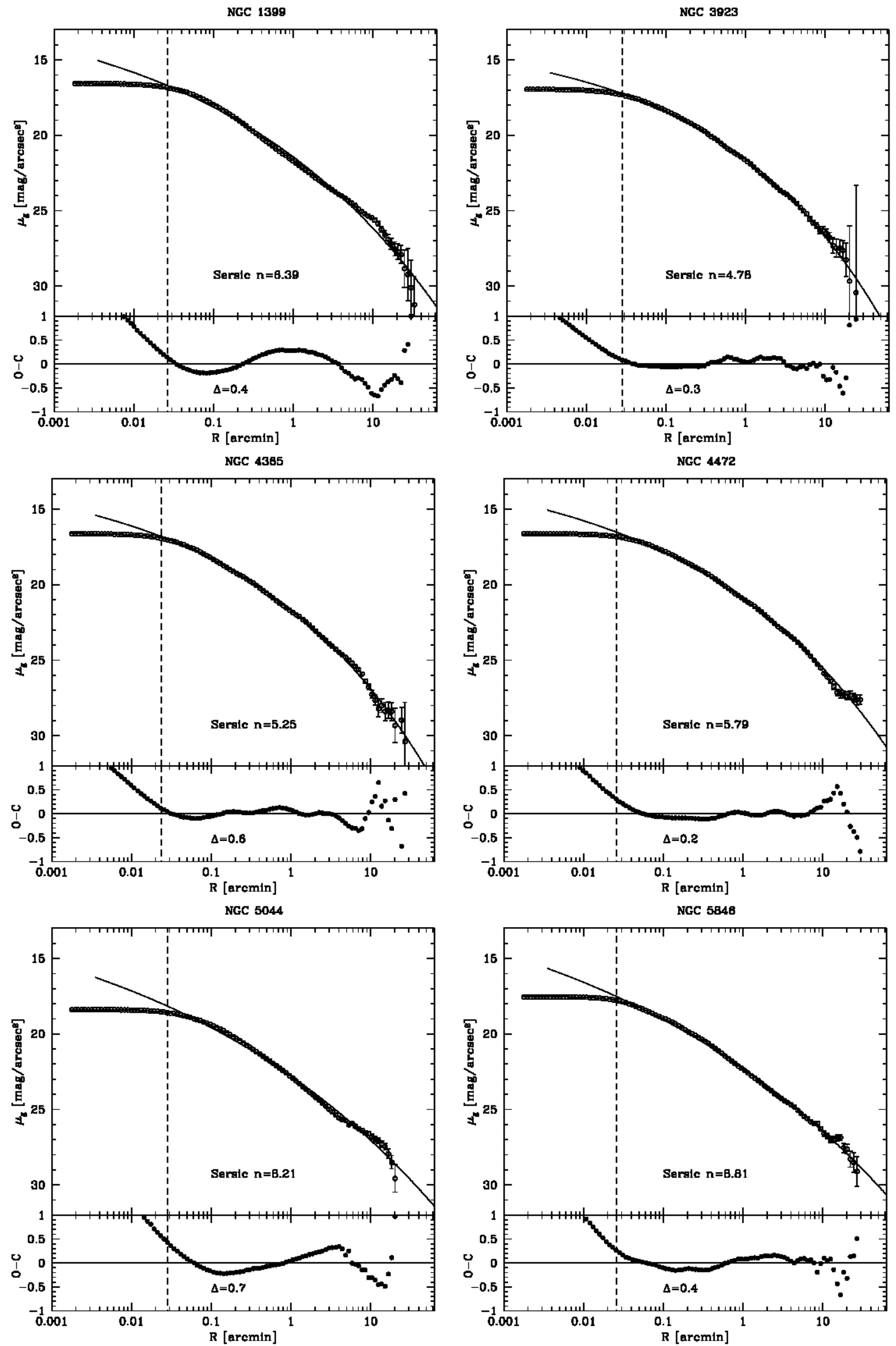

Fig. D.6. VST $g$-band profiles of NGC 1399, NGC 3923, NGC 4365, NGC 4472, NGC 5044, and NGC 5846 plotted on a logarithmic scale. The black line is a fit to the surface brightness profiles with a single Sérsic law. The dashed line indicates the core of the galaxy $(\sim 1.5 \times F W H M)$, which has been excluded in the fit. 

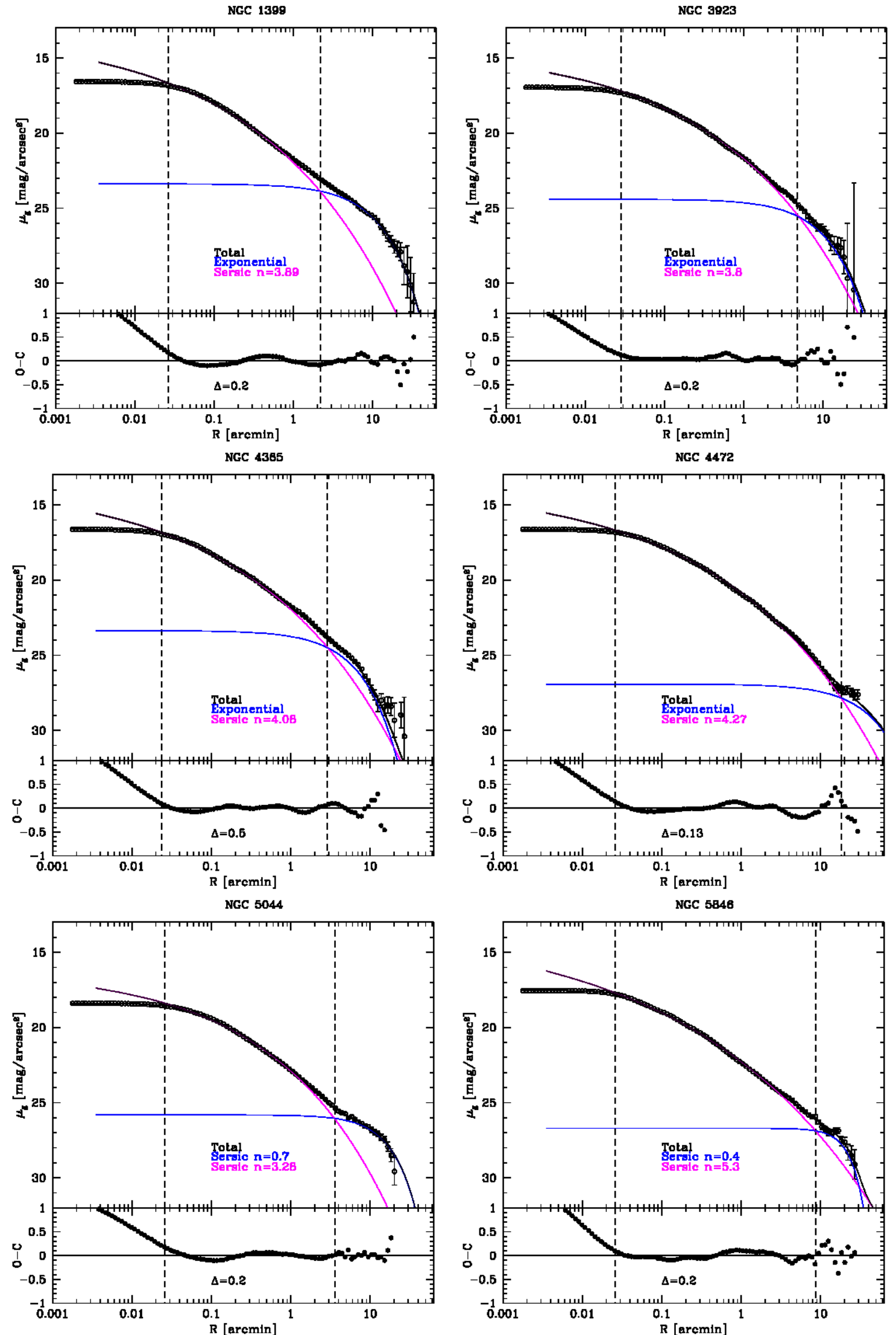

Fig. D.7. VST $g$-band profiles of NGC 1399, NGC 3923, NGC 4365, NGC 4472, NGC 5044, and NGC 5846 plotted on a logarithmic scale. The blue line is a fit to the outer regions with an exponential component, for NGC 1399, NGC 3923, NGC 4365, and NGC 4472, and with a Sérsic component for NGC 5044 and NGC 5846. The magenta line is a fit to the inner regions with a Sersic profile, and the black line is the sum of the components in each fit. The dashed lines indicate the core of the galaxy $(1.5 \times F W H M)$, which was excluded in the fit, and the transition point between the two components, respectively. 

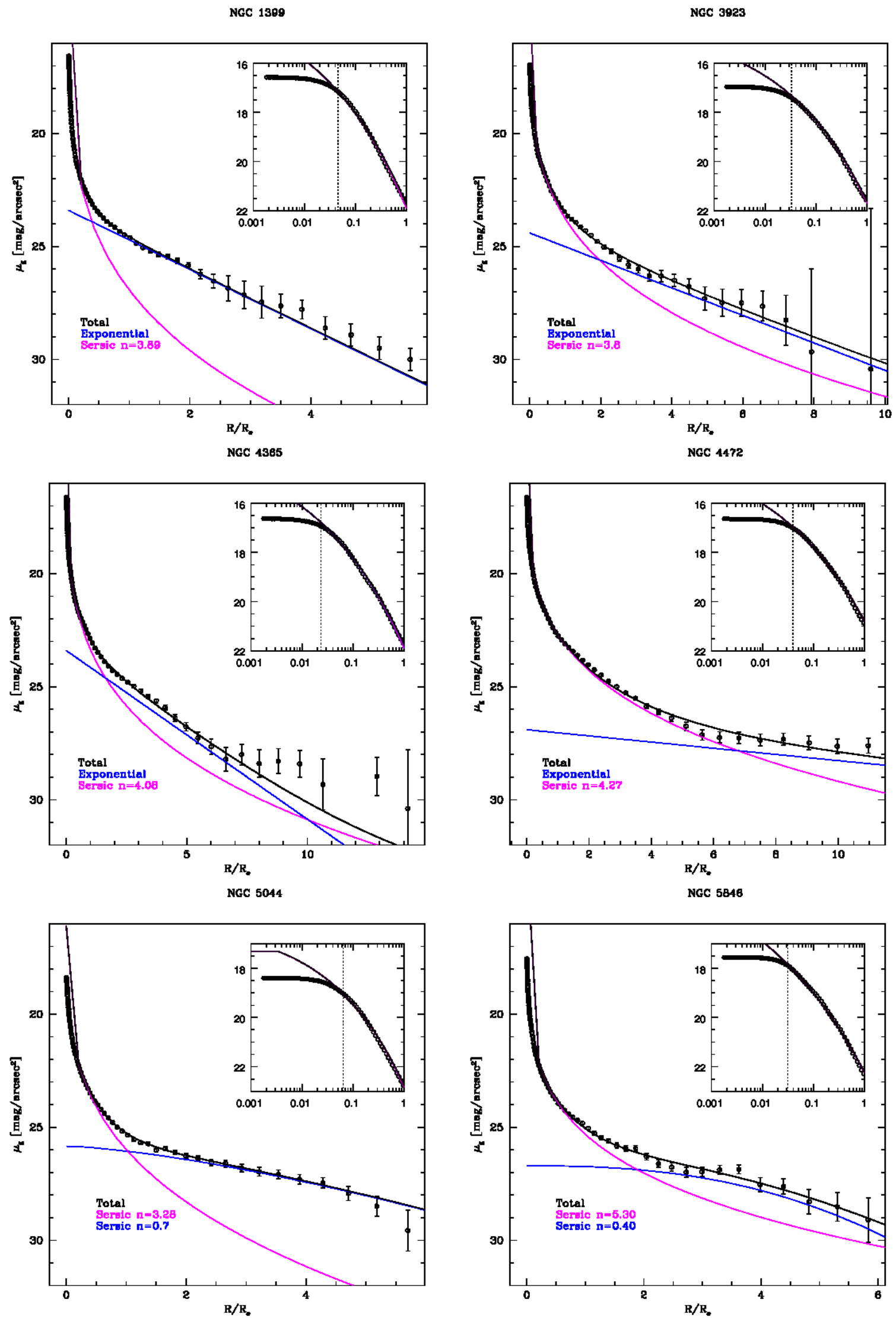

Fig. D.8. Same as Fig. D.7, in linear scale as a function of $R / R_{\mathrm{e}}$. In the small boxes, the enlarged portions of the inner regions in a log radius scale are shown and the dashed lines indicate the cores of the galaxies $(\sim 1.5 \times F W H M)$, which have been excluded from the fit. 

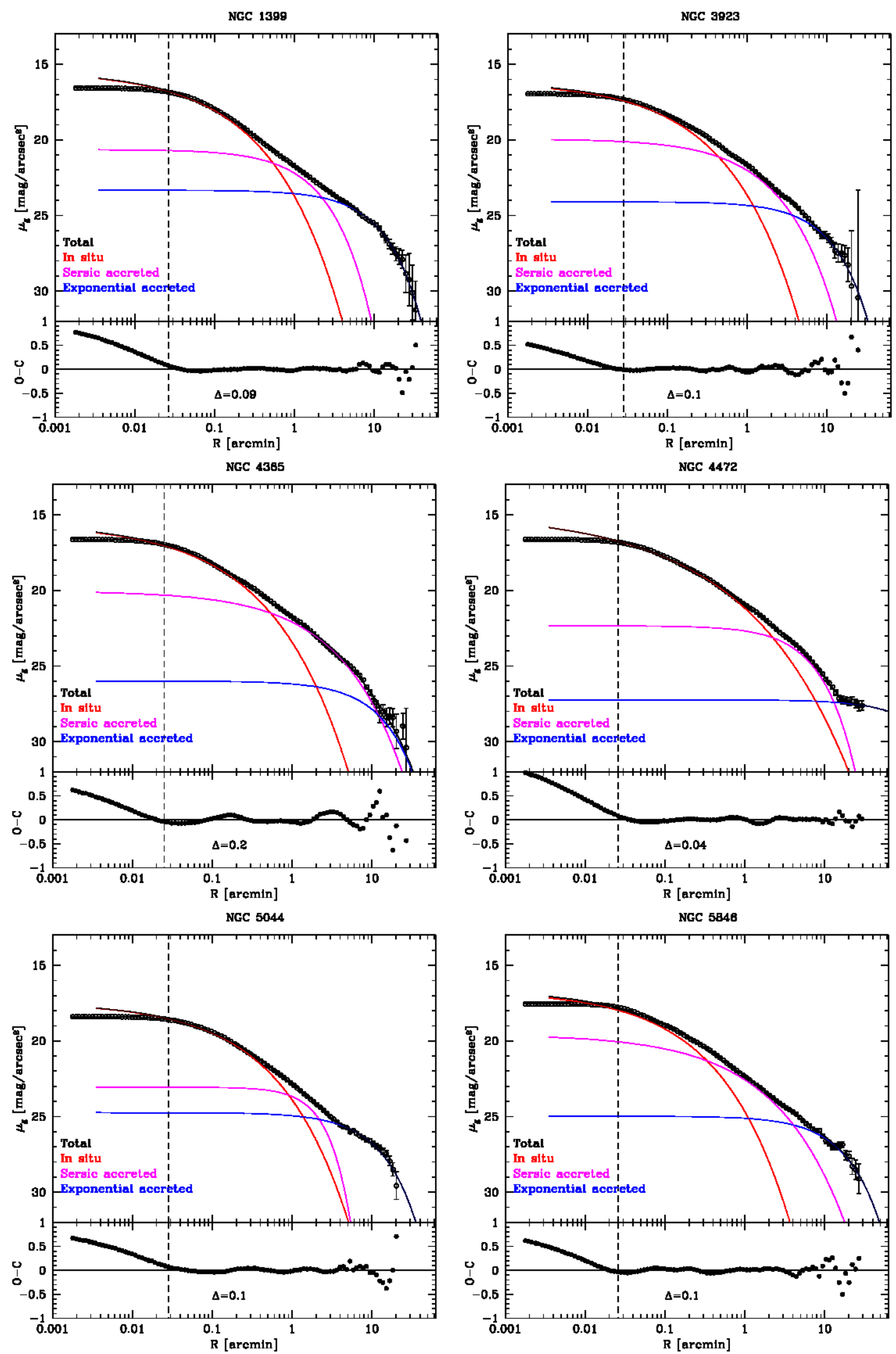

Fig. D.9. VST $g$-band profiles of NGC 1399, NGC 3923, NGC 4365, NGC 4472, NGC 5044, and NGC 57.546, fitted with a three-component model motivated by the predictions of theoretical simulations. 